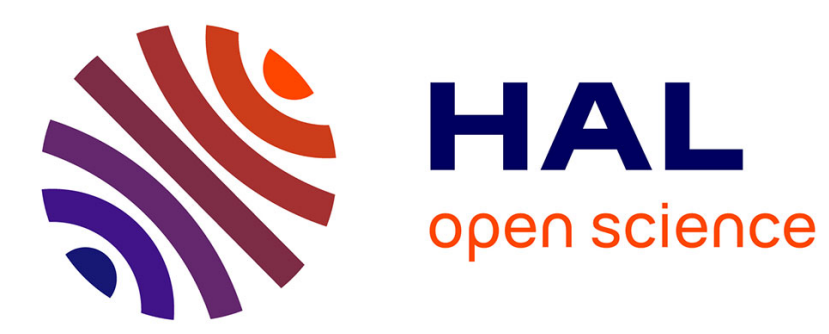

\title{
A review of two models for tolerance analysis of an assembly: jacobian and torsor
}

Wilma Polini, Massimiliano Marziale

\section{To cite this version:}

Wilma Polini, Massimiliano Marziale. A review of two models for tolerance analysis of an assembly: jacobian and torsor. International Journal of Computer Integrated Manufacturing, 2010, 24 (01), pp.74-86. 10.1080/0951192X.2010.531286 . hal-00652136

\section{HAL Id: hal-00652136 \\ https://hal.science/hal-00652136}

Submitted on 15 Dec 2011

HAL is a multi-disciplinary open access archive for the deposit and dissemination of scientific research documents, whether they are published or not. The documents may come from teaching and research institutions in France or abroad, or from public or private research centers.
L'archive ouverte pluridisciplinaire HAL, est destinée au dépôt et à la diffusion de documents scientifiques de niveau recherche, publiés ou non, émanant des établissements d'enseignement et de recherche français ou étrangers, des laboratoires publics ou privés. 


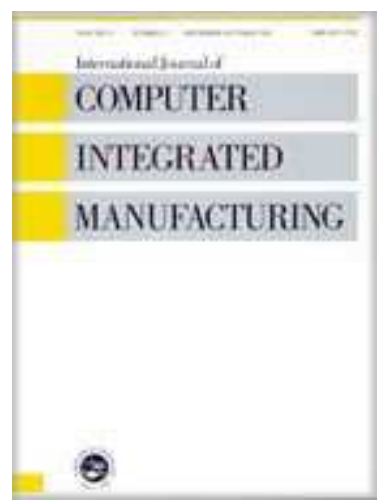

\section{A review of two models for tolerance analysis of an assembly: jacobian and torsor}

\begin{tabular}{|c|c|}
\hline Journal: & International Journal of Computer Integrated Manufacturing \\
\hline Manuscript ID: & TCIM-2009-IJCIM-0119.R2 \\
\hline Manuscript Type: & Original Manuscript \\
\hline $\begin{array}{r}\text { Date Submitted by the } \\
\text { Author: }\end{array}$ & 04-Oct-2010 \\
\hline Complete List of Authors: & $\begin{array}{l}\text { Polini, Wilma; Università degli Studi di Cassino, Dipartimento di } \\
\text { Ingegneria Industriale } \\
\text { Marziale, Massimiliano; Università degli Studi di Cassino, } \\
\text { Dipartimento di Ingegneria Industriale }\end{array}$ \\
\hline Keywords: & TOLERANCE ANALYSIS, TOLERANCING \\
\hline Keywords (user): & jacobian model, torsor model \\
\hline
\end{tabular}

\section{SCHOLARONE Manuscripts}


A review of two models for tolerance analysis of an assembly: Jacobian and Torsor

Massimiliano Marziale, Wilma Polini

Università degli Studi di Cassino, via G. di Biasio 43, 03043 Cassino, Italy

Ph. +3907762993679, Fax +3907762993546, polini@unicas.it 


\section{A review of two models for tolerance analysis of an assembly: Jacobian and Torsor}

The dimensional and geometrical variations of each part of an assembly have to be limited by tolerances able to ensure both a standardized production and a certain level of quality, which is defined by satisfying functional requirements. The appropriate allocation of tolerances among the different parts of an assembly is the fundamental tool to ensure assemblies that work rightly at lower costs. Therefore, there is a strong need to develop a tolerance analysis to satisfy the requirements of the assembly by the tolerances imposed on the single parts. This tool has to be based on a mathematical model able to evaluate the cumulative effect of the single tolerances.

Actually there are some different models used or proposed by the literature to make the tolerance analysis of an assembly constituted by rigid parts, but none of them is completely and univocally accepted. Some authors focus their attention on the solution of single problems found in these models or in their practical application in computer-aided tolerancing systems. But none of them has done an objective and complete comparison among them, analyzing the advantages and the weakness and furnishing a criterion for their choice and application.

This paper briefly introduces two of the main models for tolerance analysis, the jacobian and the torsor. These models are briefly described and then compared showing their analogies and differences. The evolution of these two models, known as unified jacobian-torsor model, is presented too.

Keywords: tolerance analysis, jacobian model, torsor model

\section{Introduction}

There is a strong need for industries to produce high precision assemblies at lower costs.

Therefore, there is a strong need to use tolerance analysis to predict the effects of the tolerances that have been assigned to the components of an assembly on the functional requirements of the assembly itself. The aim of the tolerance analysis is to study the accumulation of dimensional and/or geometric variations resulting from a stack of dimensions and tolerances. The results of the analysis are meaningfully conditioned by the adopted mathematical model.

Some are the models proposed by the literature to carry out a tolerance analysis of an assembly constituted by rigid parts. The foremost works are found in Requicha that introduced the mathematical definition of the tolerance's semantic (Requicha 1983, Requicha 1993) and proposed a solid offset approach for this purpose. Since then, a lot of models are 
proposed by the literature: the vector loop uses vectors to represent relevant dimensions in an assembly (Chase et al. 1995, Chase et al. 1996, Gao et al. 1998); the variational uses homogeneous transformation matrix to represent the variability of an assembly due to tolerances and assembly constraints (Martino et al. 1989, Boyer et al. 1991, Gupta et al. 1993); the matrix uses displacement matrix to describe any roto-translational variation a feature may be subjected to (Salomons et al. 1996, Desrochers et al. 1997); the tolerance-map uses hypothetical solid including points in $\mathrm{n}$-dimensions which represent all possible variations of a feature or an assembly (Davidson et al. 2002, Mujezinovic et al. 2004, Ameta et al. 2007, Shen et al. 2008, Singh et al. 2009); the jacobian uses an approach derived from the description of kinematic chains in robotics to formulated the displacement matrices; and the torsor uses screw parameters to model three dimensional tolerance zones. In the literature, some studies compare these models for tolerance analysis by dealing with their general features (Hong et al. 2002, Shen et al. 2005). Other studies compare the main Computer Aided Tolerancing softwares that implement some of the models of the tolerance analysis (Salomons et al. 1998, Prisco et al. 2002); but these studies focus the attention only on the general features of the considered models. Moreover, there does not exist a paper that compares the different analytical methods on the basis of a case study that underlines in a clear way all the advantages and the weakness; therefore, no guidelines exist to select the method more appropriate to the specific aims.

The purpose of this work is to analyse two of the most significant models for tolerance analysis of rigid-parts assembly: the model called jacobian and the model called torsor. The comparison of the models starts from their application to a case study. Dimensional and geometrical tolerances have been considered as part of stack-up functions. The worst and the statistical approaches have been taken into account. The application of the Envelope Principle (ASME 1994) and of the Independence Principle (ISO 1985) has been deeply investigated. Finally, the evolution of these two models, called unified jacobian-torsor model, is presented too; it should overcome the limits of the two compared models. 
Two further works of the authors compare the other main developed models of the literature: the matrix and the vector loop models (Marziale and Polini 2009) and the variational models (Marziale and Polini 2010).

Section 2 gives an overall explanation of the jacobian and the torsor models. Section 3 gives a comprehensive comparison of the two models by means of a case study that is characterized by $2 \mathrm{D}$ tolerance stack-up functions. It offers some guidelines for those who will have to make the choice too. Finally, Section 4 presents the evolution of the two models, the unified jacobian-torsor, that seems to overcome some of the limits of the jacobian and torsor models.

\section{Tolerance analysis models}

\section{Jacobian model}

Jacobian model uses pairs of Functional Elements (FEs) to represent both the dimensions and the variations of an assembly. The Functional Elements pairs are arranged in chains representing those dimensions that stack together to determine the resultant assembly Functional Requirement (FR). This model is based on the idea that the tolerances allow small linear and angular dispersions to a functional element with respect to its nominal position, orientation and form (this last is usually neglected). These dispersions are expressed by pairs of Functional Elements used to model a stack around the Functional Requirement of an assembly. Two types of FE pairs in a tolerance stack can be distinguished: internal pairs, which are pairs of the same part, and kinematic pairs, which are pairs of different parts in contact; however these two types of elements are schematized in the same way.

Based on the results sought in the kinematic of robot, a generic dispersion in a pair of Functional Elements can be expressed by a set of six virtual joints and coordinate frames (see Figure 1). In this way the spatial relationship relating the position and the orientation of the terminal FE, expressed in the Datum Reference Frame (DRF) of the base FE, is given by (Lafond et al. 1999, Laperrière et al. 1999): 


$$
\begin{aligned}
& T_{0}^{6}=
\end{aligned}
$$

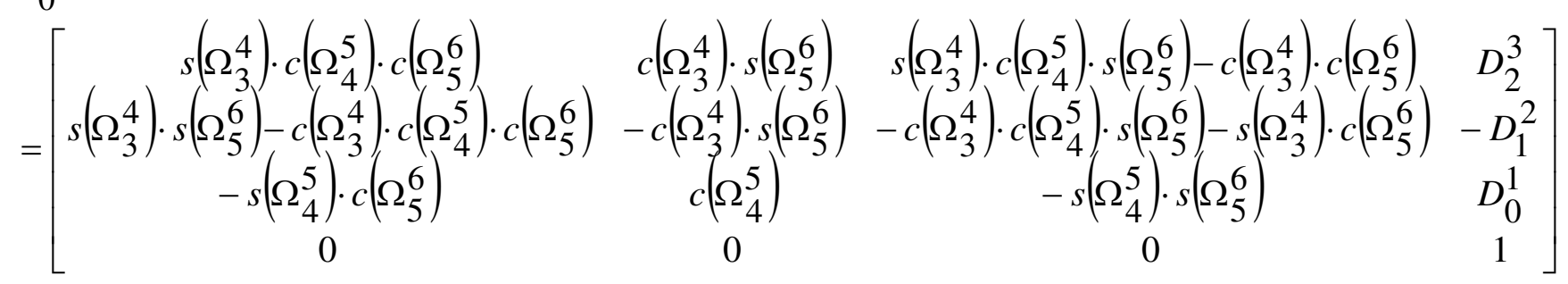

where $D_{i}^{j}$ is the variable quantifying the translation from frame $\mathrm{i}$ to frame $\mathrm{j}$ (linear dimension of the part), and $\Omega_{i}^{j}$ is the variable quantifying the rotation from frame $\mathrm{i}$ to frame $\mathrm{j}$ (angular dimension of the part). It follows that the relative position and orientation of any FE in the stack of "n" elements, with respect to the DRF of the base FE, as a result of both nominal dimensions and degrees of freedom of the FEs involved in the tolerance stack, is given by:

$$
T_{0}^{6 n}=T_{0}^{6} \cdot T_{6}^{12} \cdot \ldots \cdot T_{6 n-6}^{6 n}
$$

where " $n$ " represents the total number of FE pairs (both internal and kinematic) involved in the tolerance stack. The global deviation of the FR, expressed in DRF of the first feature $\mathrm{R}_{0}$, can be expressed as:

$$
\left[\begin{array}{c}
\delta \vec{s} \\
\delta \vec{\alpha}
\end{array}\right]=\left[\left[J_{1} J_{2} \ldots J_{6}\right]_{F E_{i}} \ldots\left[J_{6 n-5} J_{\left.6 n-4 \ldots J_{6 n}\right]_{F E_{n}}}\right] \cdot\left[\begin{array}{c}
\vec{\delta}_{F E_{i}} \\
\cdot \\
\cdot \\
\vec{\delta}_{F E_{n}}
\end{array}\right]\right.
$$

where $\delta \vec{s}$ is the vector of the 3 small translations of the considered point, expressed in the DRF of the first feature $\mathrm{R}_{0} ; \delta \vec{\alpha}$ is the vector of the 3 small rotations of the considered point, expressed in the DRF of the first feature $\mathrm{R}_{0} ;\left[J_{1} J_{2} \ldots J_{6}\right]_{F E_{i}}$ is the $6 \mathrm{x} 6$ Jacobian matrix associated with the FE of the $\mathrm{i}^{\text {th }} \mathrm{FE}$ pair (internal or kinematic) to which the tolerances are applied, with $\mathrm{i}=1$ to $\mathrm{n} ; \vec{\delta}_{F E_{i}}$ is the 6-vector of small dispersions associated with the FE of the $\mathrm{i}^{\text {th }} \mathrm{FE}$ pair (internal or kinematic) to which the tolerances are applied, expressed in the local DRF, with $\mathrm{i}=1$ to $\mathrm{n}$.

For small rotational virtual joints, the $\mathrm{i}^{\text {th }}$ column of the Jacobian matrix $J_{i}$ is computed as: 


$$
J_{i}=\left[\begin{array}{c}
\vec{z}_{0}^{i-1} x\left(d_{0}^{n}-d_{0}^{i-1}\right) \\
\vec{z}_{0}^{i-1}
\end{array}\right]
$$

while for small translational virtual joints, there is no contribution to small rotational displacements of the point of interest and the $\mathrm{i}^{\text {th }}$ column of the Jacobian matrix $J_{i}$ is computed simply as:

$$
J_{i}=\left[\begin{array}{c}
\vec{z}_{0}^{i-1} \\
\overrightarrow{0}
\end{array}\right]
$$

where $\vec{z}_{0}^{i-1}$ is the $3^{\text {rd }}$ column of $T_{0}^{i-1}$; and $\vec{d}_{0}^{i-1}$ is the last column of $T_{0}^{i-1}$. Equation (3) relates the small dispersions of the assembly FR to the virtual joints of the FE pairs, that form the stack, by a Jacobian matrix that is easily to evaluate from the nominal position of the features. Despite this advantage, the virtual joints displacements, that are the independent variable in equation (3), are difficult to relate to the tolerances applied to the assembly components.

To better understand this method, the basic steps to build a stack of Functional Element's pairs and to carry out a tolerance analysis are given below:

(1) Identify the assembly Functional Requirement and the Functional Element's pairs the first step is to identify the Functional Requirement of the assembly and the Functional Elements that are involved in the stack. Therefore, the Functional Elements are arranged in consecutive pairs to form the Functional Requirement stack.

(2) Locate the virtual joints and the DRFs of each FE - the next step is to locate the virtual joints and the DRFs of each FE. Therefore, the transformation matrix (1) is calculated for each FEs.

(3) Create the chain and the Jacobian matrix - The Functional Requirement chain (2) is evaluated by means of the transformation matrix evaluated in the step 2. Therefore, the Jacobian matrix of the assembly Functional Requirement is evaluated too by equations (4) and (5).

(4) Derive the Functional Requirement equation - once calculated the Jacobian matrix of the Functional Requirement, the dispersion of the virtual joints, due to the applied 
tolerances, have to be derived. Therefore, the displacement of the Functional Requirement may be evaluated and its equation (i.e. the stack-up function) may be formalized.

(5) The stack-up function may be solved by means of the usual methods of the literature (Whitney 2004) for both the worst case or statistical case approaches.

A fundamental consideration about this model is that it is based on both a Technologically and Topologically Related Surfaces (TTRS) (Clément et al. 1998) and a positional tolerancing (Clément et al. 1994) criteria; therefore, the tolerances of a generic drawing need to be translated according with the previously described criteria before performing the analysis. Moreover, this model may be applied to assemblies involving joints which makes a linear structure among the parts (stack-up function, see Figure 2a), while joints which makes a complex structure among the parts (network function, see Figure 2b) are not supported.

\section{Torsor model}

The torsor model uses screw parameters to model three dimensional tolerance zones (Desrochers et al. 1999, Legoff et al. 1999). Each actual surface of a part is modelled by a substitute surface. A substitute surface is a surface that has the shape of the nominal surface and it is used to model the actual surface. A substitute surface is characterized by a set of screw parameters which are the deviation of the substitute surface from the nominal one. For each of the seven types of tolerance zone, there are the correspondent screw parameters obtained by annulling the ones that leave the surface invariant in its local frame. The obtained screw parameters are arranged in a particular mathematical operator called "torsor". Considering a generic feature, if $u_{A}, v_{A}, w_{A}$ are the translation parameters of the point $\mathrm{A}$, and $\alpha, \beta, \gamma$ are the rotation angles (considerated small) as regards to the nominal position, the torsor of point $\mathrm{A}$ is given by:

$$
T=\left\{\begin{array}{ll}
\alpha & u_{A} \\
\beta & v_{A} \\
\gamma & w_{A}
\end{array}\right\}_{R}
$$


where $\mathrm{R}$ is the DRF where the screw parameters are evaluated. Once known the torsor of point $\mathrm{A}$, the torsor of point $\mathrm{B}$ may be evaluated as:

$$
T=\left\{\begin{array}{ll}
\alpha & u_{B} \\
\beta & v_{B} \\
\gamma & w_{B}
\end{array}\right\}_{R}
$$

where:

$$
\left\{\begin{array}{l}
u_{B} \\
v_{B} \\
w_{B}
\end{array}\right\}=\left\{\begin{array}{l}
u_{A} \\
v_{A} \\
w_{A}
\end{array}\right\}+\left[\begin{array}{ccc}
0 & -\gamma & \beta \\
\gamma & 0 & -\alpha \\
-\beta & \alpha & 0
\end{array}\right] \cdot\left\{\begin{array}{l}
A B_{x} \\
A B_{y} \\
A B_{z}
\end{array}\right\}=\left\{\begin{array}{l}
u_{A}-\gamma \cdot A B_{y}+\beta \cdot A B_{z} \\
v_{A}+\gamma \cdot A B_{x}-\alpha \cdot A B_{z} \\
w_{A}-\beta \cdot A B_{x}+\alpha \cdot A B_{y}
\end{array}\right\}
$$

where $\mathrm{AB}_{\mathrm{x}}, \mathrm{AB}_{\mathrm{y}}$ and $\mathrm{AB}_{\mathrm{z}}$ qre the vectors of the distance between points $\mathrm{A}$ and $\mathrm{B}$ along the $\mathrm{x}$, $\mathrm{y}$ and $\mathrm{z}$ axes respectively.

To model the interaction between the parts of an assembly, three kinds of torsors (or Small Displacement Torsor SDT) may be defined (Ballot et al. 1995, Ballot et al. 1997): a part SDT for each part of the mechanism $(\mathrm{A}, \mathrm{B}, \ldots)$ to model the displacement allowed to the part; a deviation SDT for each surface $(\mathrm{A} 1, \mathrm{~A} 2, \ldots)$ of each part to model the geometrical variations of the surface; a gap SDT between two surfaces linking two parts to model the joint properties.

Therefore, a union of the set of SDTs that are involved at the joints is used in order to obtain the global behaviour of the mechanism. This may be bone by considering that, with the worst case approach, the cumulative effect of a simple chain of n-elements is simply expressed by adding the single components of the torsors:

$$
T_{0 / n}=T_{0 / 1}+T_{1 / 2}+\ldots+T_{n-1 / n}
$$

(it is to observe that to compute this sum is necessary that the components of all the single torsors are referred to the same point B and in the same datum reference frame R).

The basic steps of the torsor model are (Villeneuve et al. 2001):

(1) Identify the elements of the parts and the relations among them - the first step is to identify the elements of the parts and the relations among them; these information are 
usually reported in a Surfaces Graph. The Functional Requirements and the stacks to relate these FRs are identified too.

(2) Define the parameters of the mechanism - a deviation torsor has to be associated to each surface of the parts, therefore, a characterization of the global SDT of each part has to be done. The shape of the gap torsor, that is associated to each joint according to the functional conditions required by the assembly, has to be defined too.

(3) Computate the cumulative effect of the torsors involved in each stack in order to evaluate the Functional Requirements by equation (9).

Finally, some fundamental considerations are needed. The first is that this model is developed under the hypothesis to use the TTRS and the positional tolerancing criteria. The second is that the solution of stacks arranged in a network is not completely developed in spite of the different works produced in the last years (Teissandier et al. 2007, Franciosa et al. 2009). The third is that the torsor components assume a double meaning. In a first approach the small displacement torsor components are simple parameters and they are computed by means of common algebraic rules. An example of this approach is in (Legoff et al. 1999) where a tolerance problem involving network functions is solved. In the second approach the small displacement torsor components are admissible intervals according with the applied tolerance ranges. An example of this approach is in (Teissandier et al. 1999). The first approach gives a solution to the tolerance analysis problems very similar to the other approaches of the literature; while the second approach may theoretically relate the variability range of the Functional Requirements of the assembly to the assigned tolerance ranges. However, this second approach needs to compute the small displacement torsor components, that are intervals, by means of the arithmetic by interval, that is not fully developed yet. In the following we make reference only to this second approach, since its potentialities seem more interesting in tolerance analysis. 


\section{Models comparison}

\section{Case Study}

To compare the two models previously described the case study shown in Figure 3 has been used. The two-dimensional geometry of the example assembly is made of a rectangular box containing two disk-shaped parts. The width $g$ of the gap between the top disk and the upper surface of the box is assumed as the functional requirement to be investigated by the analysis. Goal of the tolerance analysis problem is to identify the tolerance stack-up function that defines the variability of $g$, and describes it as a function of the geometries and tolerances of the components involved in the assembly.

Tolerance analysis is based on the dimensional and geometrical tolerances illustrated in Figure 4. The example is adapted from a real-life industrial application and properly simplified to make it easier to be presented and discussed in this context. The applied tolerancing scheme, which may not appear as entirely rigorous under the viewpoint of a strict application of standardized tolerancing rules, is directly derived from the current practice adopted for the actual industrial product.

The case study is representative of all the main aspects and critical issues involved in a typical tolerance analysis problem, and it is simple enough to allow for applying a simplified manual computation procedure for obtaining the extreme values of the gap $g$, for the special case where only dimensional tolerances are considered. The manual computation is based on searching for the worst-case conditions, i.e. the combinations of part dimensions that give origin to the maximum and minimum gap values; since no geometric tolerances are considered, part geometries are assumed at nominal states.

The maximum value of the gap has been calculated by considering the maximum height and width of the box, together with the minimum value of the radius of the disks:

$$
g_{\max _{\operatorname{dim}}}=80.5-19.95-\sqrt{(19.95 \cdot 2)^{2}-(504-19.95-19.95)^{2}}-19.95=2.1064 \mathrm{~mm}
$$

In the same way the minimum value of the gap is due to: 
$g_{\text {min }_{\operatorname{dim}}}=79.5-20.05-\sqrt{(20.05 \cdot 2)^{2}-(49.80-20.05-20.05)^{2}}-20.05=0.4909 m m$

The variability of the gap is the difference between the maximum or the minimum values and the nominal one:

$$
\begin{aligned}
& \Delta g_{\operatorname{dim}_{1}}=+\left(g_{\max _{\operatorname{dim}}}-g_{N}\right)=(2.1064-1.2702) \cong+0.84 m m \\
& \Delta g_{\operatorname{dim}_{2}}=-\left(g_{N}-g_{\min _{\operatorname{dim}}}\right)=-(1.2702-0.4909) \cong-0.78 \mathrm{~mm}
\end{aligned}
$$

Although operating on a simplified problem (geometrical tolerances are neglected) the manual computation of the gap boundary values provides a useful support for the quantitative comparison of the five methods, at least when they are applied by considering dimensional tolerances only. The manually-obtained, extreme gap values will be used as reference values later on, then the results of the five methods will be discussed.

\section{Jacobian model solution}

\section{Dimensional tolerances only}

The jacobian model of the case study is made under the simplified hypothesis to consider as fixed at $90^{\circ}$ the orientation of the four sides of the box. This simplification is needed to avoid the network in the assembly. Indeed it is to observe (see Figure 3) that the functional requirement $g$ has to be measured between the top side of the box and the second disk. Being the second disk assembled with the sub-assembly box-disk 1, firstly the assembly between part 1 and part 2 has to be solved. Therefore, the assembly between the part 3 and the subassembly box-disk 1 has to be solved. Once indicated with $\mathrm{x}_{1}$ and $\mathrm{x}_{2}$ the dimensions of the box, with $\mathrm{x}_{3}$, and $\mathrm{x}_{4}$ the diameter of the two disks and with $\mathrm{U}_{1}, \mathrm{U}_{2}, \mathrm{U}_{3}, \mathrm{U}_{4}$ the assembly variables (see Figure 5), the simplification adopted makes it possible to directly solve the assembly problem as:

$$
\begin{aligned}
& U_{1}=x_{3} \\
& U_{2}=x_{3}
\end{aligned}
$$




$$
\begin{gathered}
U_{3}=x_{1}-x_{4} \\
U_{4}=x_{3}+\sqrt{\left(x_{3}+x_{4}\right)^{2}-\left(x_{1}-x_{3}-x_{4}\right)^{2}}
\end{gathered}
$$

Therefore, the functional requirement $g$ and the functional elements pairs of the case study are identified (Figure 5). The first functional elements pair is associated with the points $\mathrm{G}$ and $\Omega$ and it is an internal one. The second functional elements pair is associated with the points $\Omega$ and $\mathrm{O}_{1}$, while the third functional elements pair is associated with the points $\mathrm{O}_{1}$ and $\mathrm{O}_{2}$; they are both externals. The last internal functional elements pair is associated with the points $\mathrm{O}_{2}$ and $\mathrm{H}$. The required functional requirement $g$ is correspondent to the functional elements pair is associated with the points $\mathrm{G}$ and $\mathrm{H}$, and it is evaluated as the chain of the four functional elements pairs just identified. At this point, is possible for each FE to locate the virtual joints and the reference frames, and to evaluate the transformation matrices $T_{0}^{1}, T_{0}^{2}, T_{0}^{3}, \ldots, T_{0}^{24}$ with the equations (1) and (2). The matrix of the total transformation is equal to:

$$
T_{0}^{24}=\left[\begin{array}{cccc}
1 & 0 & 0 & 0 \\
0 & 1 & 0 & 0 \\
0 & 0 & 1 & -x_{2}+x_{3}+x_{4}+\sqrt{\left(x_{3}+x_{4}\right)^{2}-\left(x_{1}-x_{3}-x_{4}\right)^{2}} \\
0 & 0 & 0 & 1
\end{array}\right]
$$

Therefore, the Jacobian matrix of the functional requirement is evaluated using equations (4) and (5): 


\begin{tabular}{|c|c|c|c|c|c|}
\hline & 0 & 0 & 1 & 0 & 0 \\
\hline & 0 & -1 & 0 & 0 & 0 \\
\hline & 1 & 0 & 0 & 0 & 0 \\
\hline & 0 & $x_{1}-x_{4}$ & 0 & 0 & 0 \\
\hline & 0 & $-h$ & 0 & 1 & 0 \\
\hline & $h$ & 0 & $x_{4}-x_{1}$ & 0 & 1 \\
\hline & 0 & 0 & 1 & 0 & 0 \\
\hline & 0 & -1 & 0 & 0 & 0 \\
\hline & 1 & 0 & 0 & 0 & 0 \\
\hline & 0 & $x_{1}-x_{3}-x_{4}$ & 0 & 0 & 0 \\
\hline & 0 & $h-x_{3}$ & 0 & 1 & 0 \\
\hline$J^{T}=$ & $h-x_{3}$ & 0 & $-\left(x_{1}-x_{3}-x_{4}\right)$ & 0 & 1 \\
\hline & 0 & 0 & 1 & 0 & 0 \\
\hline & 0 & -1 & 0 & 0 & 0 \\
\hline & 1 & 0 & 0 & 0 & 0 \\
\hline & 0 & 0 & 0 & 0 & 0 \\
\hline & 0 & $-x_{4}$ & 0 & 1 & 0 \\
\hline & $x_{4}$ & 0 & 0 & 0 & 1 \\
\hline & 0 & 0 & 1 & 0 & 0 \\
\hline & 0 & -1 & 0 & 0 & 0 \\
\hline & 1 & 0 & 0 & 0 & 0 \\
\hline & 0 & 0 & 0 & 0 & 0 \\
\hline & 0 & 0 & 0 & 1 & 0 \\
\hline & 0 & 0 & 0 & 0 & 1 \\
\hline
\end{tabular}

where $h=x_{3}+x_{4}+\sqrt{\left(x_{3}+x_{4}\right)^{2}-\left(x_{1}-x_{3}-x_{4}\right)^{2}}$.

Once calculated the Jacobian matrix of the functional requirement pair, the stack-up function may be formalized considering that the requirement $\Delta g$ must be evaluated as the translation of point $\mathrm{H}$ long the $-\mathrm{Z}_{0}$ axis (Figure 5), and then as the third component of equation (3). It is:

$$
\Delta g=-\delta z_{24}=-\delta z_{0}+\delta \phi z_{5} \cdot\left(x_{1}-x_{4}\right)-\delta z_{6}+\delta \phi z_{11} \cdot\left(x_{1}-x_{3}-x_{4}\right)-\delta z_{12}-\delta z_{18}
$$

where $\delta z_{i}(\mathrm{i}=0,1,2,6,7,8,12,13,14,18,19,20)$ is the translation along the $\mathrm{i}^{\text {th }}$ axis and $\delta \phi z_{i}(\mathrm{i}=3,4,5,9,10,11,15,16,17,21,22,23)$ is the rotation around the $\mathrm{i}^{\text {th }}$ axis. This equation relates the functional requirement $\Delta g$ to the virtual joints displacements assigned to 
the functional requirement pairs; now it is necessary to relate these virtual joints displacements to the tolerances assigned on the components.

This step is the critical task of the jacobian model. However considering the simplification adopted (angles fixed) is $\delta \phi z_{5}=\delta \phi z_{11}=0$ and then:

$$
\Delta g=-\delta z_{0}-\delta z_{6}-\delta z_{12}-\delta z_{18}
$$

Moreover, with reference to Figure 5 of the four functional requirement pairs and to equations (13), and considering the nominal dimensions:

$$
\begin{aligned}
\delta z_{0} & =-\delta x_{2} \\
\delta z_{6} & =\delta x_{3} \\
\delta z_{12}=\nabla\left(\sqrt{\left(x_{3}+x_{4}\right)^{2}-\left(x_{1}-x_{3}-x_{4}\right)^{2}}\right) & =-0.2582 \cdot \delta x_{1}+1.2910 \cdot \delta x_{3}+1.2910 \cdot \delta x_{4} \\
\delta z_{18} & =\delta x_{4}
\end{aligned}
$$

and then is:

$$
\Delta g=0.2582 \cdot \delta x_{1}+\delta x_{2}-2.2910 \cdot \delta x_{3}-2.2910 \cdot \delta x_{4}
$$

Once obtained the required stack-up function, it can be solved with the usual methods of the literature. For example, for the worst case approach (Whitney 2004):

$$
\begin{aligned}
& \Delta g_{W C}= \pm \sum_{i=1}^{4}\left|S_{i}\right| \cdot t_{i}= \pm(0.2582 \cdot 0.20+0.50+2.2910 \cdot 0.05+2.2910 \cdot 0.05)= \\
& = \pm 0.7807 \cong \pm 0.78 \mathrm{~mm}
\end{aligned}
$$

while for statistical case approach:

$$
\Delta g_{S C}= \pm \sqrt{\sum_{i=1}^{4}\left(S_{i} \cdot t_{i}\right)^{2}}= \pm 0.5281 \cong \pm 0.53 \mathrm{~mm}
$$

\section{Dimensional and geometrical tolerances}

If the dimensional and the geometrical tolerances are considered, none changes as regards to the previous case, since it has been adopted the simplification to consider fixed the angles in order to avoid the network. In fact, to solve the stack-up function, it is needed to relate the virtual joints displacements to the tolerances assigned on the components. However, the form 
tolerances (the planar one applied on the bottom side of the box and the two circularities applied on the circles) do not produce any effect because in the jacobian model the features are considered with nominal shape; the other ones (the perpendicularity applied on the left side of the box, and the two parallelisms applied on the other sides of the box) cannot produce any orientation deviation, since the angles of the box are considered fixed.

Therefore, the simplification to consider fixed the angles of the box, due to the need to avoid network, causes the assigned geometrical tolerances do not produce any effects and the results are the same as the previous case where are considered only dimensional tolerances.

Moreover, the application of the Envelope Principle or of the Independence Principle does not produce any effect for the jacobian model.

\section{Torsor model solution}

\section{Dimensional tolerances only}

The required functional characteristic $g$ has to be evaluated by considering that there is a network among the components of the assembly. At the state of the art, the solution of parallel chains through the torsor method is not completely developed. Therefore, the simplification to consider fixed the angles of the box has been used in order to avoid the network. This simplification may solve the assembly problem as showed in equations (13).

The first step of the method is to identify the elements of the parts (see Figure 6), and the relations among them; these information are reported in the surfaces graph of the case study (see Figure 7). Considering the angles of the box as fixed, the network can be solved and, therefore, the surfaces graph is simplified as showed in Figure 8. The cumulative torsor $\mathrm{G}$ is expressed as:

$$
G_{H}=-T_{1,1}+T_{1 / 3}+T_{3,1}=\left\{\begin{array}{cc}
\alpha & u_{H} \\
\beta & v_{H} \\
\gamma & \Delta g
\end{array}\right\}_{R}
$$

where $T_{l, l}$ is the torsor of feature 1 of part 1 (the box); $T_{3,1}$ is the torsor of feature 1 of part 3 (the circle 2); and $T_{1 / 3}$ is the torsor of the link between part 1 and part 3. Therefore, the functional requirement $\Delta g$ is expressed by the translation component along the global $\mathrm{Z}$ axis. 
The next step is to evaluate the components of the torsors (indeed it is enough to evaluate the third components due to translation). For the $T_{1,1}$ torsor, with reference to Figure 9 and Table 1 and considering that the case study is a 2D problem on $\mathrm{x}-\mathrm{z}$ plane (i.e. $\alpha=0, \gamma=0, v=0$ ), it is:

$$
T_{1,1}=\left\{\begin{array}{cc}
- & - \\
\beta_{1,1} & - \\
- & w_{M 1,1}
\end{array}\right\}_{R_{1,1}}
$$

where $\mathrm{M}$ is the median point of the feature. Considering that the point of interest is $\mathrm{H}$, from equation (8):

$$
\left\{\begin{array}{l}
u_{H} \\
v_{H} \\
w_{H}
\end{array}\right\}=\left\{\begin{array}{c}
- \\
- \\
w_{M 1,1}
\end{array}\right\}+\left[\begin{array}{ccc}
0 & - & \beta_{1,1} \\
- & 0 & - \\
-\beta_{1,1} & - & 0
\end{array}\right] \cdot\left\{\begin{array}{c}
5 \\
- \\
-1.2703
\end{array}\right\}=\left\{\begin{array}{c}
-1.2702 \beta_{1,1} \\
- \\
w_{M 1,1}-5 \beta_{1,1}
\end{array}\right\}
$$

and then from equation (7):

$$
T_{1,1}=\left\{\begin{array}{cc}
- & -1.2702 \beta_{1,1} \\
\beta_{1,1} & - \\
- & w_{M 1,1}-5 \beta_{1,1}
\end{array}\right\}_{R_{1,1}}
$$

This torsor (note that only three of its components are not null) is expressed in the local frame of the feature and it has to be expressed in the global reference frame R (Figure 9). In this simply case it is possible to note that the local y-axis coincides with the global Y-axis, therefore, the angle $\beta_{l, l}$ is the same too. The local $\mathrm{x}$ and $\mathrm{z}$ axes are the inverse of the global $\mathrm{X}$ and $\mathrm{Z}$ axes respectively, therefore, the correspondent translation needs to be inverted. It is:

$$
T_{1,1}=\left\{\begin{array}{cc}
- & -1.2702 \beta_{1,1} \\
\beta_{1,1} & - \\
- & -\left(w_{M 1,1}-5 \beta_{1,1}\right)
\end{array}\right\}_{R}
$$

Considering the simplification of considering fixed the angles of the box, $\beta_{l, 1}=0$ and therefore:

$$
T_{1,1}=\left\{\begin{array}{cc}
- & - \\
0 & - \\
- & -w_{M 1,1}
\end{array}\right\}_{R}
$$


This torsor has constrained by considering that the extreme points $\mathrm{F}$ and $\mathrm{Q}$ remain into the tolerance zone. It results:

$$
T_{1,1}=\left\{\begin{array}{cc}
- & - \\
0 & -\left[\left(t_{1,1}+t_{2}\right) / 2,+\left(t_{1,1}+t_{2}\right) / 2\right] \\
- & -[-\}_{R}
\end{array}\right\}^{-}
$$

where $t_{1,1}$ is the thickness of the tolerance zone of $\mathrm{S}_{1,1}$, and $t_{2}$ is the dimensional tolerance of the dimension $x_{2}$. Note that this torsor shows the admissible range of variations of the small displacements associated to the feature. In the same way the torsor $T_{3,1}$ of feature 1 of part 3 may be computed; it results:

$$
T_{3,1}=\left\{\begin{array}{ll}
- & {\left[-t_{4} / 2,+t_{4} / 2\right]} \\
0 & {\left[-t_{4} / 2,+t^{t_{4} / 2}\right]}
\end{array}\right\}_{R}
$$

where $t_{4}$ is the dimensional tolerance of the dimension $x_{4}$.

The evaluation of the torsor linking part 1 and part $3\left(T_{1 / 3}\right)$ is very difficult because it needs the solution of the networks among the components of the assembly. By adopting the simplification that the angles of the box are fixed, it is possible to solve this problem as the sum of two terms:

$$
T_{1 / 3}=T_{1 / 2}+T_{2 / 3}
$$

The first term $\left(T_{1 / 2}\right)$ is the torsor of the link between part 1 and part 2 . With reference to Figure 9 it is:

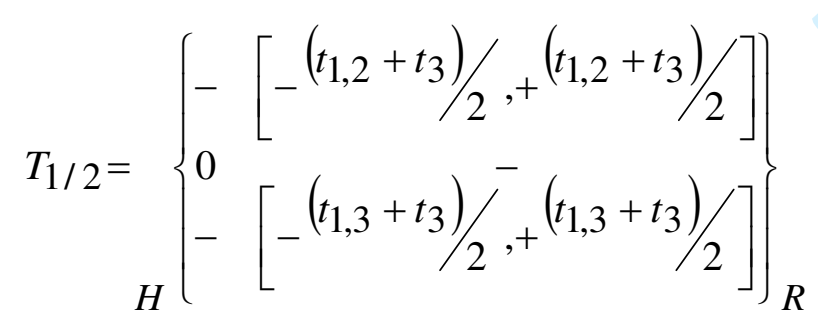

The second term $\left(T_{2 / 3}\right)$ is the torsor of the link between part $1+2$ and part 3 . With reference to Figure 9 it is: 


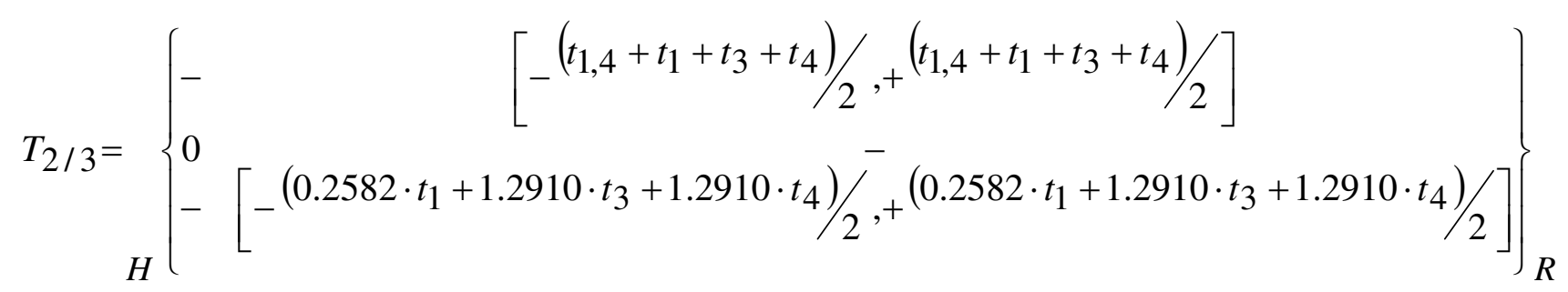

where $t_{1,2}, t_{1,3}$, and $t_{1,4}$ are the thicknesses of the tolerance zones of $\mathrm{S}_{1,2}, \mathrm{~S}_{1,3}$ and $\mathrm{S}_{1,4}$; and $t_{1}$, $t_{3}$, and $t_{4}$ are the tolerances on the dimensions $x_{1}, x_{3}$ and $x_{4}$. Therefore, the functional requirement is:

$$
\Delta g= \pm\left(0.2582 \cdot t_{1}+t_{2}+2.2910 \cdot t_{3}+2.2910 \cdot t_{4}+t_{11}+t_{13}\right) / 2
$$

Now, it is necessary to relate the thicknesses of each tolerance zone assigned to each feature to the tolerances required on the components. This is another critical step of the torsor model. However, under the simplified hypothesis adopted (i.e. fixed angles of the box) and by considering only dimensional tolerances, it may have:

$$
\begin{aligned}
t_{11}=t_{12} & =t_{13}=t_{14}=0 \mathrm{~mm} \\
t_{1} & =0.40 \mathrm{~mm} \\
t_{2} & =1.00 \mathrm{~mm} \\
t_{3} & =0.10 \mathrm{~mm} \\
t_{4} & =0.10 \mathrm{~mm}
\end{aligned}
$$

And, therefore, the functional requirement in the worst case approach is:

$$
\Delta g_{W C}= \pm(0.2582 \cdot 0.40+1.00+2.2910 \cdot 0.10+2.2910 \cdot 0.10) / 2= \pm 0.7807 \cong \pm 0.78 \mathrm{~mm}
$$

as obtained with the jacobian approach. It may be added that the torsor method does not allow to evaluate the results due to a statistical approach, since the torsor's components are considered the extreme possible intervals of the small displacements, and this is not compatible with the statistical approach where a probably density function is to each parameter. 


\section{Dimensional and geometrical tolerances}

If the dimensional and the geometrical tolerances are considered, equation (30) is still valid (under the hypothesis of fixed angles of the box), and it is always needed to relate the thickness of the tolerance zones to the tolerances required on the components. Moreover, by using the simplification to consider fixed the angles of the box, none changes as regards the case of only dimensional tolerances. Therefore, the simplification to consider fixed the angles of the box causes the geometrical tolerances do not have effects on the results of the case study.

Moreover, the application of the Envelope Principle or the Independence Principle does not produce any effect for the torsor model too.

\section{Comparison}

Table 1 shows the results due to the application of the two considered models to the same case study. If only the dimensional tolerances are applied, the worst case approach gives small under estimated results of about $4 \%$, when compared with the geometrical exact solution. This is probably due to the same way the dimensional tolerances are schematize (i.e. the first datum is nominal, the variability due to the dimensional tolerance is considered applied only on one of the two features delimiting the dimension). If the geometrical tolerances are applied too, the worst case approach gives the same result, since it has been adopted the simplification to consider fixed the angles of the box in order to avoid the network. In fact, to solve the stack-up function, it is needed to relate the virtual joints displacements to the tolerances assigned on the components. However, the form tolerances (the planar one applied on the bottom side of the box and the two circularities applied on the circles) do not produce any effect because in the jacobian model the features are considered with nominal shape; the other ones (the perpendicularity applied on the left side of the box, and the two parallelisms applied on the other sides of the box) cannot produce any orientation 
deviation, since the angles of the box are considered fixed. The statistical approach gives similar results, when only dimensional tolerances or both dimensional and geometrical tolerances are applied. In this case the variability range is smaller than that of the worst case approach, as it is foreseen.

The jacobian model has the advantage, as regards the torsor model, to be able to perform the analysis by both the worst case and the statistical approaches. Moreover, the jacobian model allows to evaluate the jacobian matrix from the nominal conditions and, therefore, it is possible to directly relate the displacements of the functional requirements to the virtual joints displacements. Another advantage of the jacobian model is that it uses the usual algebraic rules to evaluate the displacements of the functional requirements, while the torsor model needs intervals algebraic rules which are much complex; this aspect is fundamental to approach the solution of the network functions which need to be developed for both the models.

Despite these advantages of the jacobian model, its virtual joints displacements are difficult to relate to the tolerances applied on the components. This step is critical for the torsor model too, but it seems easier to approach, despite with this second model the computation of the displacement components of the points of interest in the same global datum reference frame is very difficult and it has to be developed completely yet.

\section{Unified Jacobian-Torsor model}

As just underlined, the jacobian model takes its best advantage in the simplicity to evaluate the jacobian matrix from the nominal conditions; equations (4) and (5). This makes it possible to directly relate the displacements of the functional requirements to the virtual joints displacements; equation (3). The solution of the network functions seems easier to approach than the torsor one too. Despite this advantage, the virtual joint displacements are difficult to relate to the tolerances applied on the components. 
The torsor model allows to easily evaluate the variability ranges of the small displacements from the tolerances applied on the components, but it is very difficult to relate these ranges to the variability ranges of the functional requirements of the assembly.

In the last years the idea of the Unified Jacobian-Torsor model has been presented in order to evaluate the virtual joint displacements from the tolerances applied on the components by the torsors and, therefore, to relate the displacements of the functional requirements to the virtual joint displacements by the jacobian matrix (Laperrière et al. 2002, Desrochers et al. 2003); it is theoretically possible since the deviations are usually small and the equations may be linearized. The proposed unified model expands the functionalities of the jacobian model under two important aspects (Ghie et al. 2003). First, the punctual small displacement variables of the former jacobian formulation are now considered as intervals formulated and solved using interval-base arithmetic. The equations describing the bounds within which the feature is permitted to move, which are the constraint equations of the torsor formulation, are applied on the unified model. Second, some of the small displacement variables used in the model are eliminated due to the invariant nature of the movements they generate with respect to the toleranced feature. This standard result of the torsor formulation is applied to the unified model. The effect of this is to significantly reduce the unified model size. This new model enables to perform tolerance analysis and tolerance synthesis (Laperrière et al. 2002) or to redesign the assembly tolerances (Ghie et al. 2007). The Unified Jacobian-Torsor model has been developed for deterministic (worst-case) computer aided-tolerancing. Recently, the same set of interval-based deterministic equations has been applied to a statistical context (Ghie et al. 2010) and the model has been used to develop a method for obtaining the functional requirement cost for product (Ghie 2009).

\section{Conclusions}

This paper firstly makes a brief review of two state of the art tolerance analysis models for rigid-parts assembly, the jacobian and the torsor. The two models are compared, in order to 
highlight the advantages and the weakness of each model, on the basis of the experimental results and the information available from the literature.

The two considered models have some common limits. The first two deal with the assembly cycle: the two models have not developed yet an approach to consider functional requirement functions arranged in a network and to correctly represent the coupling with clearance between two parts. The third deals with the representation of the tolerances applied on the assembly's components: the two models does not give a complete correspondence among the model variables (displacements) and the part's tolerances. In other words the translation of the part's tolerances into model variables does not satisfy the standards (ASME or ISO). The last deals with the form deviations and the Independence Principle: the two models do not allow to consider form deviations (the features are considered with nominal shape) and to apply the Independence and/or the Envelope Rule to different tolerances of the same parts.

However, the jacobian model has the advantage to directly relate the displacements of the functional requirements to the virtual joints displacements by the evaluation of the jacobian matrix from the nominal conditions. Moreover, it can be used to perform both a worst case and a statistical case approaches.

The torsor model has an easily evaluation of the variability ranges of the small displacements from the tolerances applied to the components.

The experimental results of the case study show how both the jacobian and the torsor models give the same underestimated result; this is due to the adopted simplification to consider fixed the angles of the box.

To overcome these limits in the last years a new model called Unified Jacobian-Torsor Model has been proposed, but it is still object of research.

Further researches include the definition of a new and original model able to overcome the limits that have been highlighted in this work. 


\section{Acknowledgements}

This research was partially funded by the Italian Ministry of University and Research.

\section{References}

Ameta, G., Davidson, J.K. and Shah, J.J., 2007. Influence of form on Frequency Distribution for 1-D clearance which is generated from Tolerance-Maps. Proceedings of $10^{\text {th }}$ CIRP Conference on Computer Aided Tolerancing, March $21^{\text {st }}-23^{\text {rd }}$, Erlangen, Germany.

ASME Standard, 1994. ASME Y14.5M-1994: Dimensioning and tolerancing. American Society of Mechanical Engineers, New York.

Ballot, E. and Bourdet, P., 1995. Geometrical behaviour laws for computer aided tolerancing. Proceedings of the $4^{\text {th }}$ CIRP Seminar on Computer Aided Tolerancing, University of Tokyo, April 1995, 143-154.

Ballot, E.and Bourdet P., 1997. A computational method for the consequences of geometric errors in mechanisms. Proceedings of the $5^{\text {th }}$ CIRP Seminar on Computer Aided Tolerancing, Toronto, April $27^{\text {th }}-29^{\text {th }}, 137-148$.

Boyer, M. and Stewart, N.F., 1991. Modeling spaces for toleranced objects. International Journal of Robotics Research, 10(5), 470-582.

Chase, K.W., Gao, J. and Magleby, S.P., 1995. General 2-D tolerance analysis of mechanical assemblies with small kinematic adjustments. Journal of Design and Manufacturing, 5(4), 263-274.

Chase, K.W., Gao, J., Magleby, S.P. and Sorensen, C.D., 1996. Including geometric feature variations in tolerance analysis of mechanical assemblies. IIE Transactions, 28(10), 795807.

Clément, A., Rivière, A. and Temmerman, M., 1994. Cotation tridimensionelle des systèmes mécaniques, théorie \& pratique. Cachan, France.

Clément, A., Riviére, A., Serré, P. and Valade, C., 1998. The TTRSs: 13 Constraints for Dimensioning and Tolerancing . In: H. A. ElMaraghy, ed. Geometric Design Tolerancing: Theories, Standards and Applications. London, Chapman \& Hall, 123-131. 
Davidson, J.K., Mujezinovic, A. and Shah, J.J., 2002. A new mathematical model for geometric tolerances as applied to round faces. ASME Transactions, Journal of Mechanical Design, 124, 609-622.

Desrochers, A. and Rivière, A., 1997. A Matrix Approach to the Representation of Tolerance Zones and Clearances. The International Journal of Advanced Manufacturing Technology, $13,630-636$.

Desrochers, A., 1999. Modeling three dimensional tolerance zones using screw parameters. Proceedings DETC $25^{\text {th }}$ Design Automation Conference, September $12^{\text {th }}-15^{\text {th }}$, Las Vegas, Nevada, USA.

Desrochers, A., Ghie, W. and Laperriére, L., 2003. Application of a Unified Jacobian-Torsor Model for tolerance analysis. Journal of Computing and Information Science in Engineering, 3, 2-14.

Franciosa, P., Gerbino, S. and Patalano, S., 2009. Modelling and Simulation of Assembly Constraints in Tolerance Analysis of Rigid Part Assemlies. Proceedings of $11^{\text {th }}$ CIRP Conference on Computer Aided Tolerancing, March $26^{\text {th }}-27^{\text {th }}$, Annecy, France.

Gao, J., Chase, K.W. and Magleby, S.P., 1998. Generalized 3-D Tolerance Analysis of mechanical assemblies with small kinematic adjustments. IIE Transactions, 30, 367-377.

Ghie, W., Laperrière, L. and Desrochers, A., 2003. A Unified Jacobian-Torsor model for analysis in Computer Aided Tolerancing. In: Recent advances in integrated design and manufacturing in Mechanical Engineering, Gogu G., Coutellier D., Chedmail P., Ray P. eds, Kluwer Academic Publishers, The Netherlands, 63-72.

Ghie, W., Laperrière, L. and Desrochers, A., 2007. Re-design of Mechanical Assemblies using the Unified Jacobian- Torsor Model for Tolerance Analysis. In: Models for Computer Aided Tolerancing in Design and Manufacturing, Davidson J.K. ed., Springer, 95-104.

Ghie, W., 2009. Functional requirement cost for product using Jacobian-Torsor model. Proceedings of $11^{\text {th }}$ CIRP International Conference on Computer Aided Tolerancing, Annecy, France, March $26^{\text {th }}-27^{\text {th }}$. 
Ghie, W., Laperrière, L. and Desrochers, A., 2010. Statistical tolerance analysis using the unified Jacobian-Torsor model. International Journal of Production Research, 48(15), 4609-4630.

Gupta, S. and Turner, J.U., 1993. Variational solid modelling for tolerance analysis. IEEE Computer Graphics and Applications, 13, 64-74.

Hong, Y. S. and Chang, T. C., 2002. A comprehensive review of tolerancing research. International Journal of Production Research, 40(11), 2425-2459.

ISO Standard, 1985. ISO 8015: Fundamental Tolerancing Principle.

Lafond, P. and Laperrière, L., 1999. Jacobian-based modeling of dispersions affecting predefined functional requirements of mechanical assemblies. Proceedings of IEEE International symposium on assembly and task planning, Porto, Portugal.

Laperrière, L. and Lafond, P., 1999. Modeling tolerances and dispersions of mechanical assemblies using virtual joints. Proceedings of ASME Design Engineering Technical Conferences, September 12-15, Las Vegas, Nevada, USA.

Laperriére, L., Ghie, W. and Desrochers, A., 2002. Statistical and Deterministic tolerance analysis and synthesis using a Unified Jacobian-Torsor Model. Annals of the $52^{\text {nd }}$ CIRP General Assembly, San Sebastian, 51(1), 417-420.

Legoff, O., Villeneuve, F. and Bourdet, P., 1999. Geometrical tolerancing in process planning: a tridimensional approach. Proc. Instn. Mech. Engrs. Part B: Journal of Engineering Manufacture, 213, 635-640.

Martino, P.M. and Gabriele, G.A., 1989. Application of variational geometry to the analysis of mechanical toleranced. Failure Prevention and Reliability, 16, 19-27.

Marziale, M. and Polini, W., 2009. A Review of two models for tolerance analysis of an assembly - Vector Loop and MATRIX. International Journal of Advanced Manufacturing Technology, 43(11-12), 1106-1123. 
Marziale, M. and Polini, W., 2010. A review of variational models for the tolerance analysis of an assembly, accepted for publication on Proceedings of the Institution of Mechanical Engineers. Part B: Journal of Engineering Manufacture.

Mujezinovic, A., Davidson, J.K. and Shah, J.J., 2004. A new mathematical model for geometric tolerances as applied to polygonal faces. ASME Transactions, Journal of Mechanical Design, 126, 504-518.

Prisco, U. and Giorleo G., 2002. Overview of Current CAT Systems. Integrated Computer Aided Engineering, 9(4), 373-397.

Requicha, A., 1983. Toward a theory of geometric tolerancing. International Journal of Robotics Research, 2(4), 45-60.

Requicha, A., 1993. Mathematical definitions of tolerance specifications. Manufacturing Review, 6(4), 269-274.

Salomons, O.W., Haalboom, F.J., Jonge Poerink, H.J., van Slooten, F., van Houten, F. J. A. M. and Kals, H. J. J., 1996. A computer aided tolerancing tool II: Tolerance analysis. Computers in Industry, 31, 175-186.

Salomons, O. W., van Houten, F. J. A. M. and Kals, H. J. J., 1998. Current Status of CAT Systems. Geometric Design Tolerancing: Theories, Standards and Applications, H.A. ElMaraghy ed., Chapman \& Hall, London, 438-452.

Shen, Z., Ameta, G., Shah, J. J. and Davidson J. K., 2005. A comparative study of tolerance analysis methods. Journal of Computing and Information Science in Engineering, 5(3), 247-256.

Shen, Z., Shah, J. J. and Davidson, J. K., 2008. Automatic generation of $\min / \mathrm{max}$ tolerance charts for tolerance analysis from CAD models. International Journal of Computer Integrated Manufacturing, 21(8), 869-884.

Singh, G., Ameta, G., Davidson, J.K. and Shah J.J., 2009. Worst-Case Tolerance Analysis of a Self-Aligning Coupling Assembly using Tolerance-Maps, Proceedings of $11^{\text {th }}$ CIRP 
International Conference on Computer Aided Tolerancing, Annecy, France, March $26^{\text {th }}$ $27^{\text {th }}$.

Teissandier, D., Couétard, Y. and Gérard, A., 1999. A computer aided tolerancing model: proportioned assembly clearance volume. Computer Aided Desing, 31, 805-817.

Teissandier, D. and Dufaure, J., 2007. Specifications of a pre and post-processing tool for a tolerancing analysis solver. Proceedings of $10^{\text {th }}$ CIRP Conference on Computer Aided Tolerancing, March $21^{\text {st }}-23^{\text {rd }}$, Erlangen, Germany.

Villeneuve, F., Legoff, O. and Landon, Y., 2001. Tolerancing for manufacturing: a threedimensional model. International Journal of Production Research, 39(8), 1625-1648.

Whitney, E. D., 2004. Mechanical Assemblies. Their Design, Manufacture and Role in Production Development. New York, Oxford University Press, ISBN 0-19-515782-6. 


\section{Figure captions}

Figure 1. Virtual joints and coordinate frames to FE pairs

Figure 2. Stack-up function a) and Network function b)

Figure 3. The case study

Figure 4. The case study with dimensional and geometrical tolerances

Figure 5. Functional Requirement and the Functional Elements pairs of the case study

Figure 6. Torsor model: elements and parts of the case study

Figure 7. Torsor model: surface graph of the case study

Figure 8. Torsor model: simplified surface graph of the case study

Figure 9. Torsor model: tolerance zones of the case study 
Table captions

Table 1. Case study results 


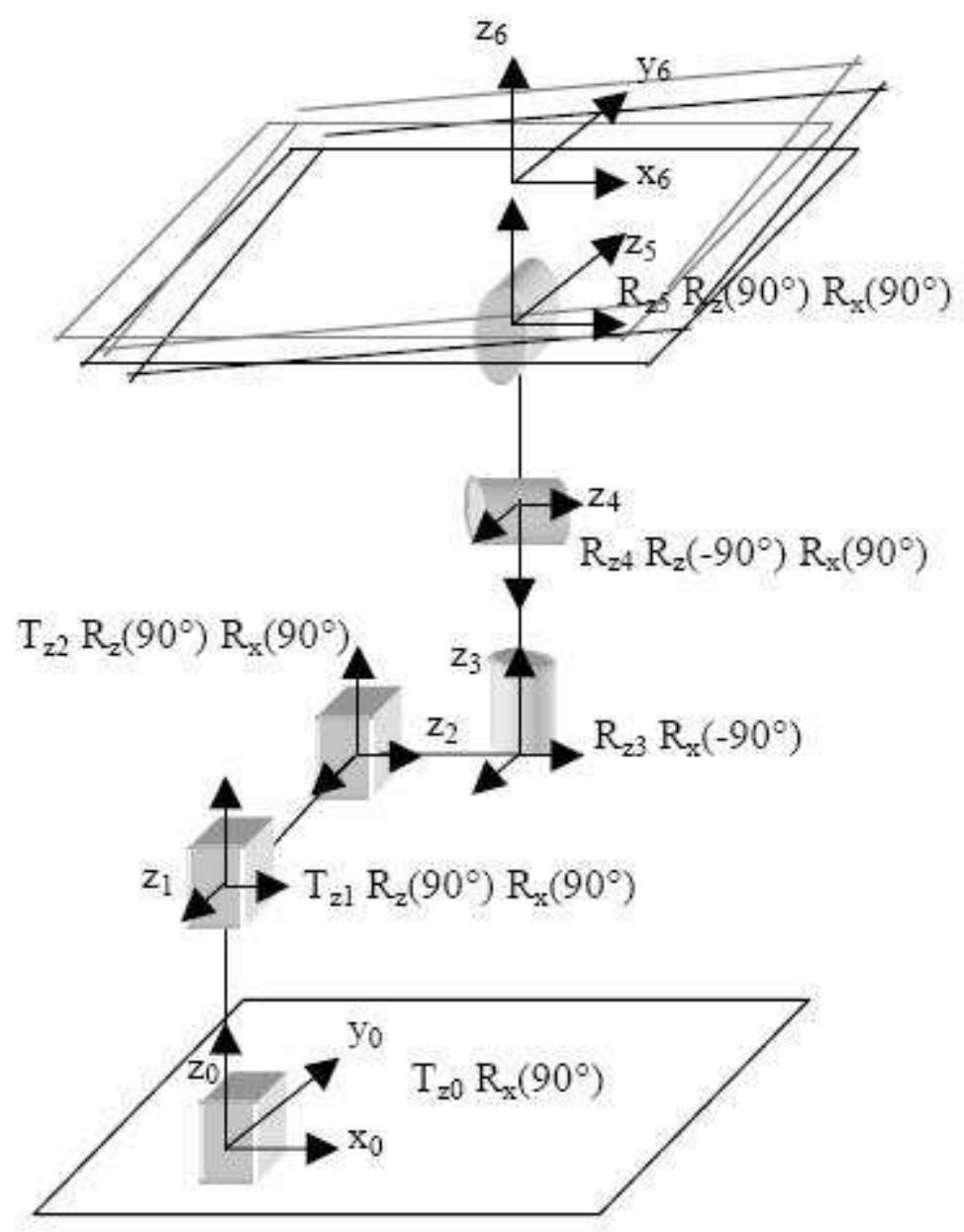

Figure 1 


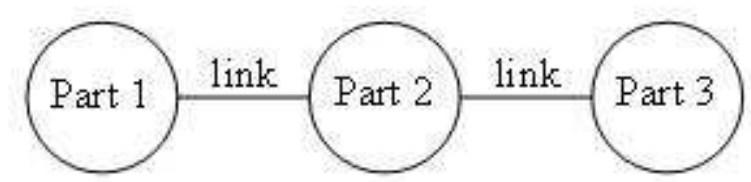

a)

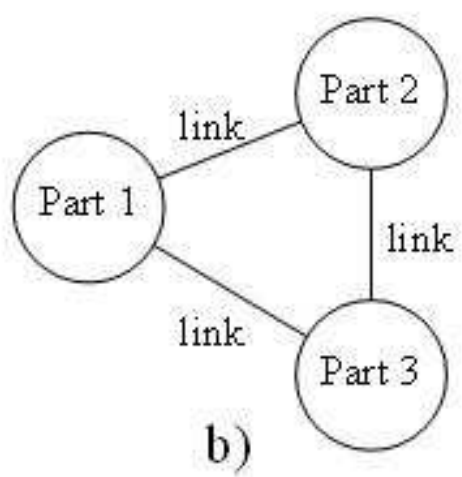

Figure 2 


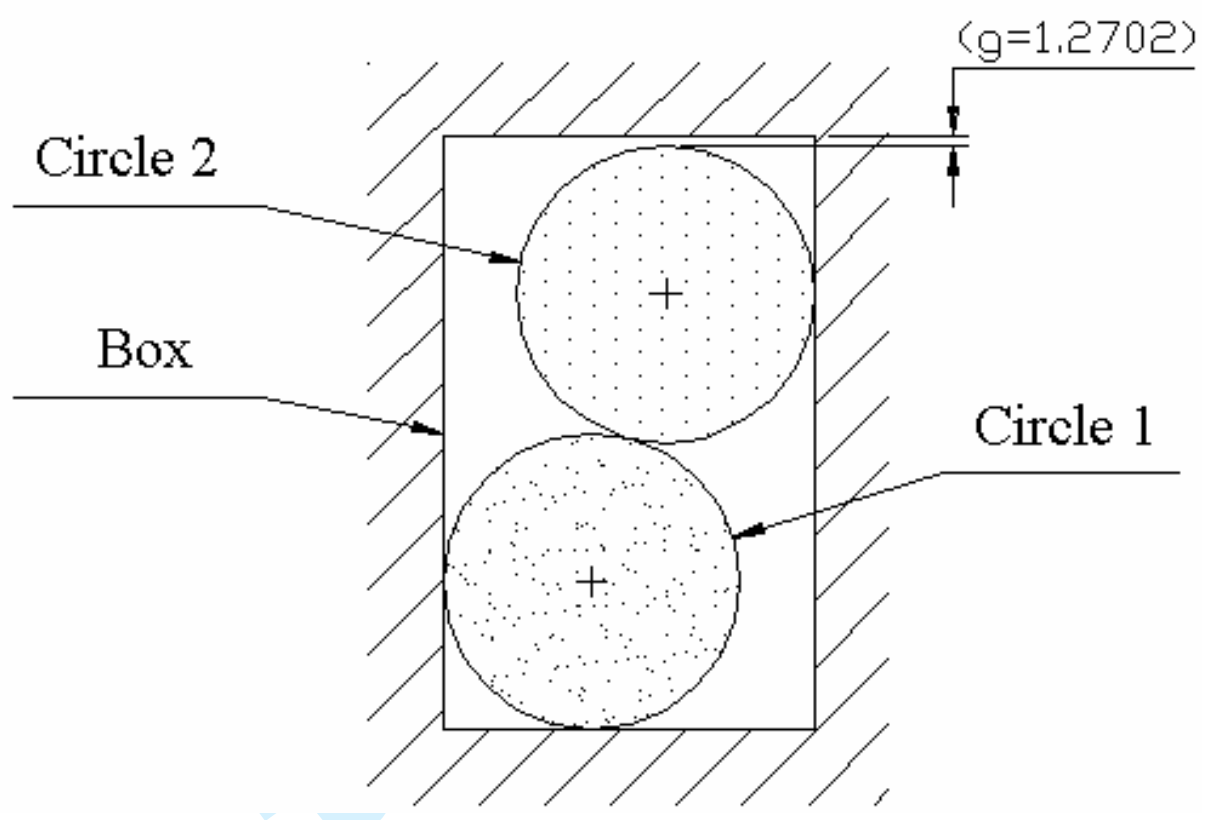

Figure 3 


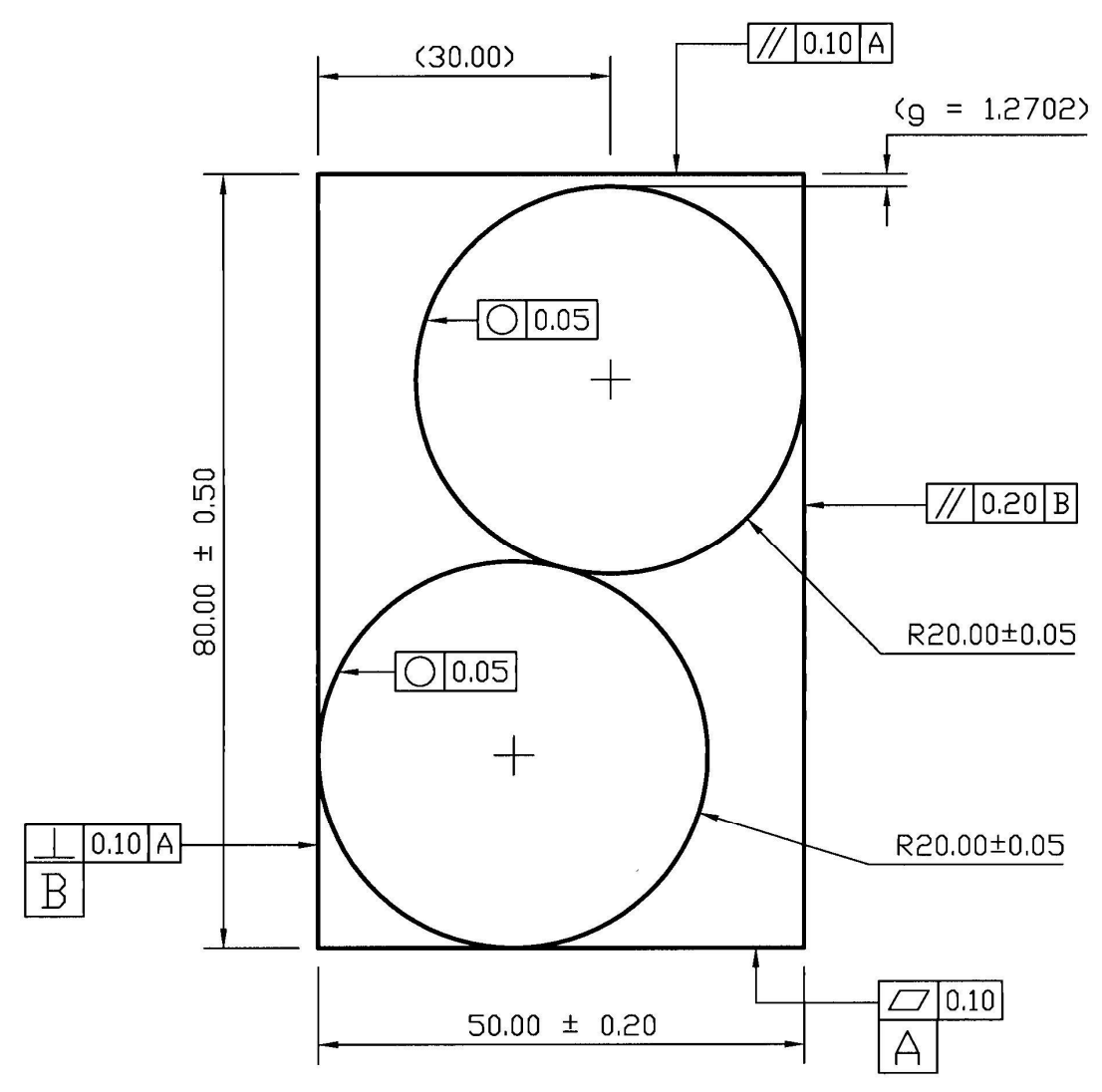

Figure 4 


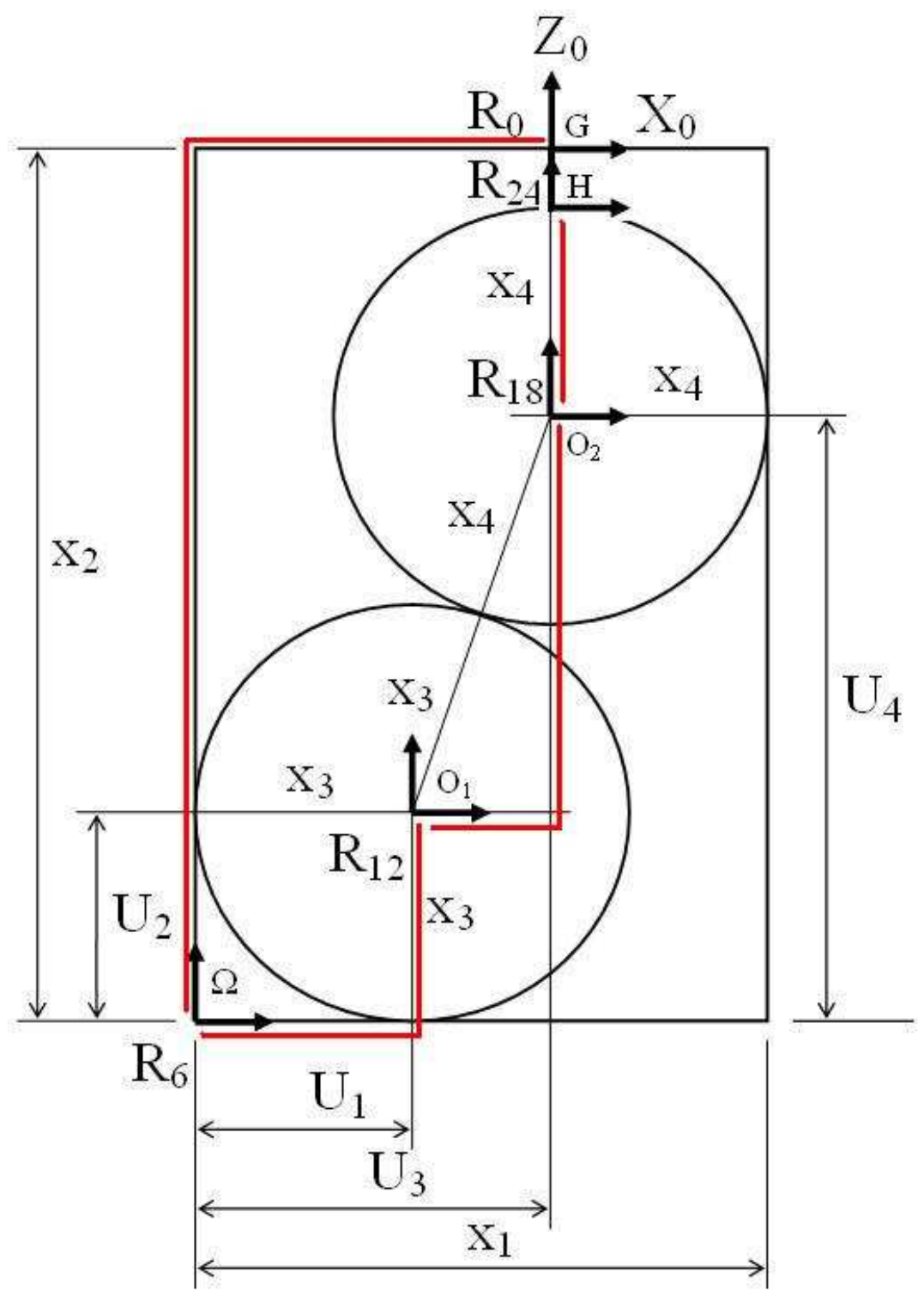

Figure 5 


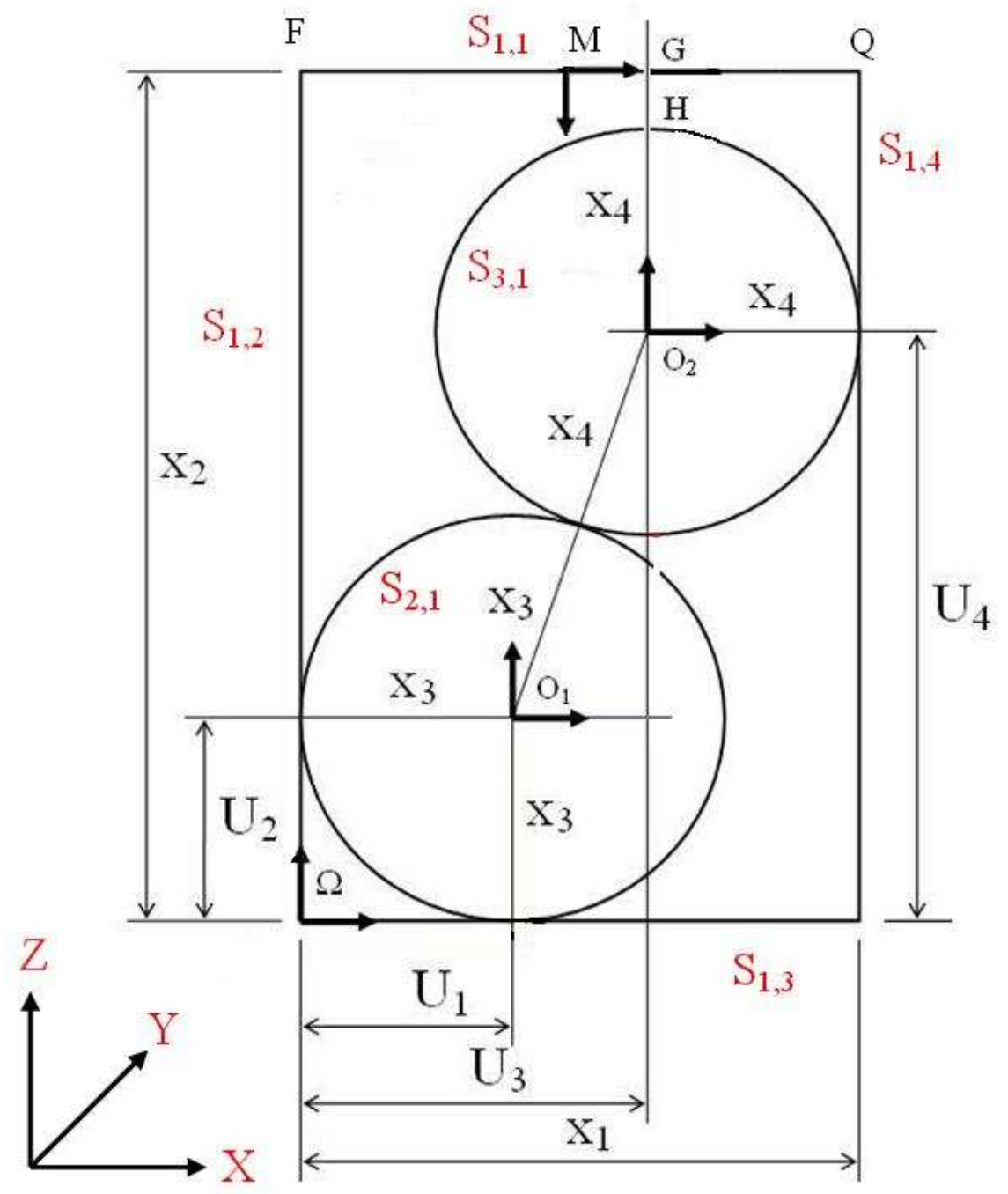

Figure 6 


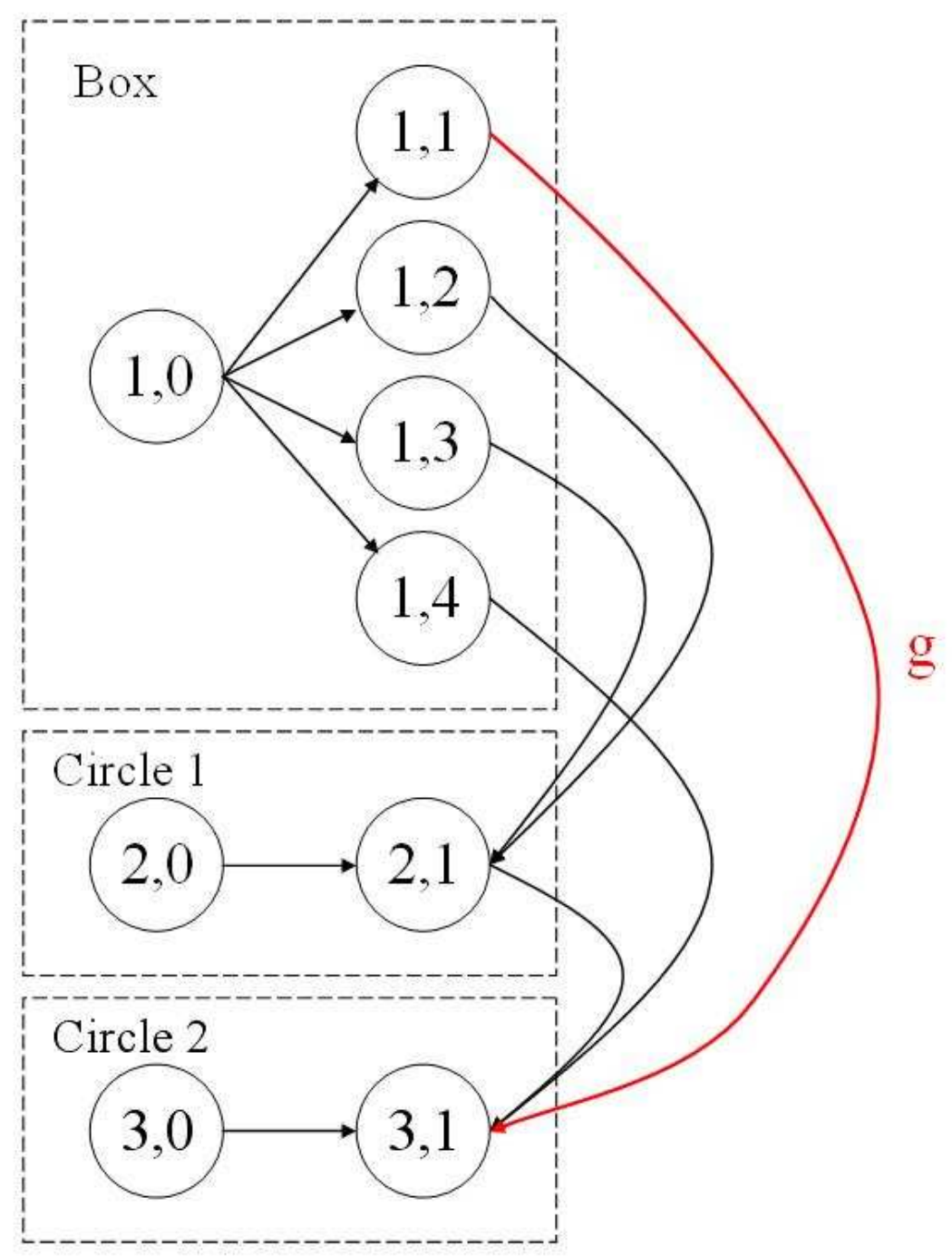

Figure 7 


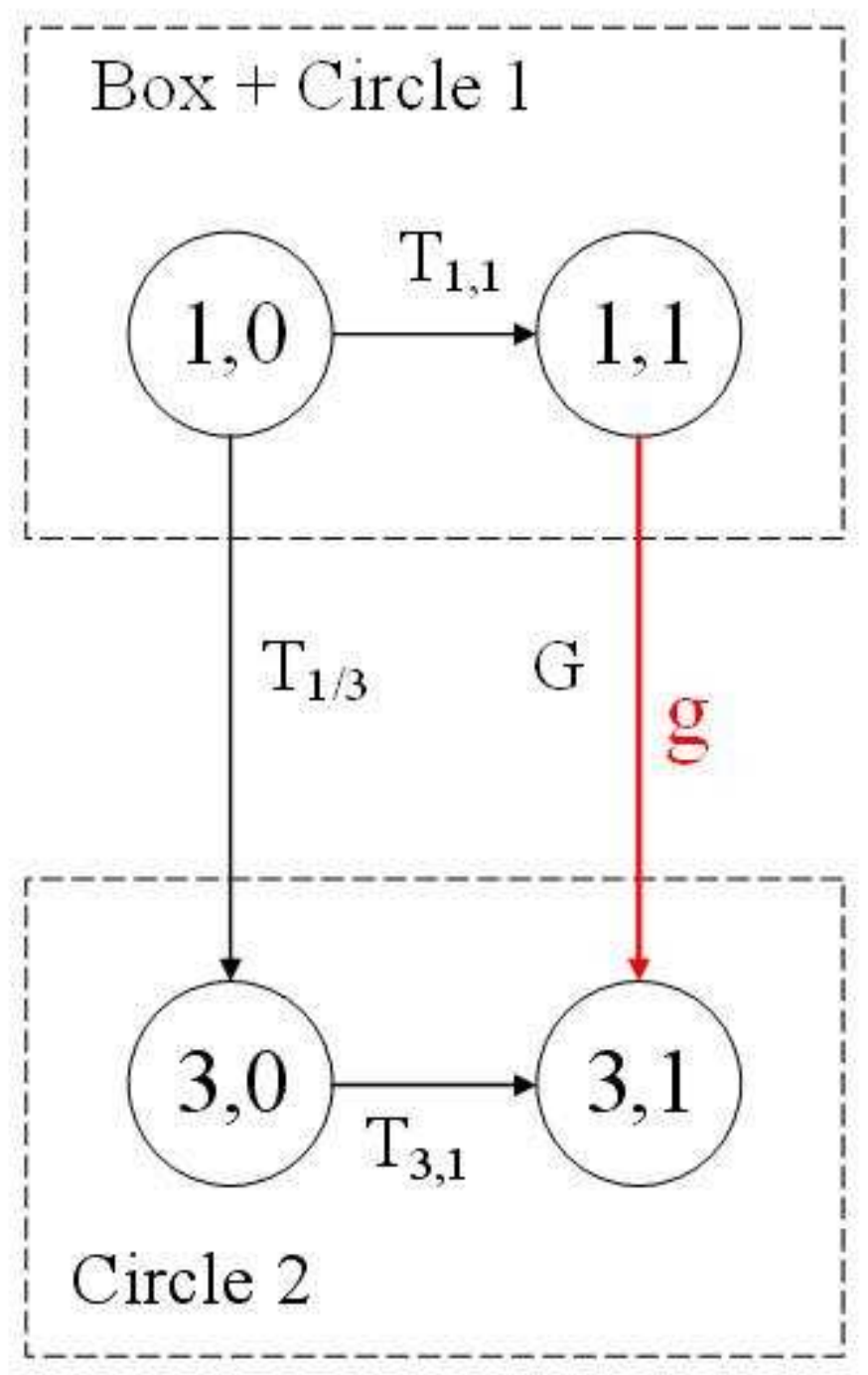

Figure 8 


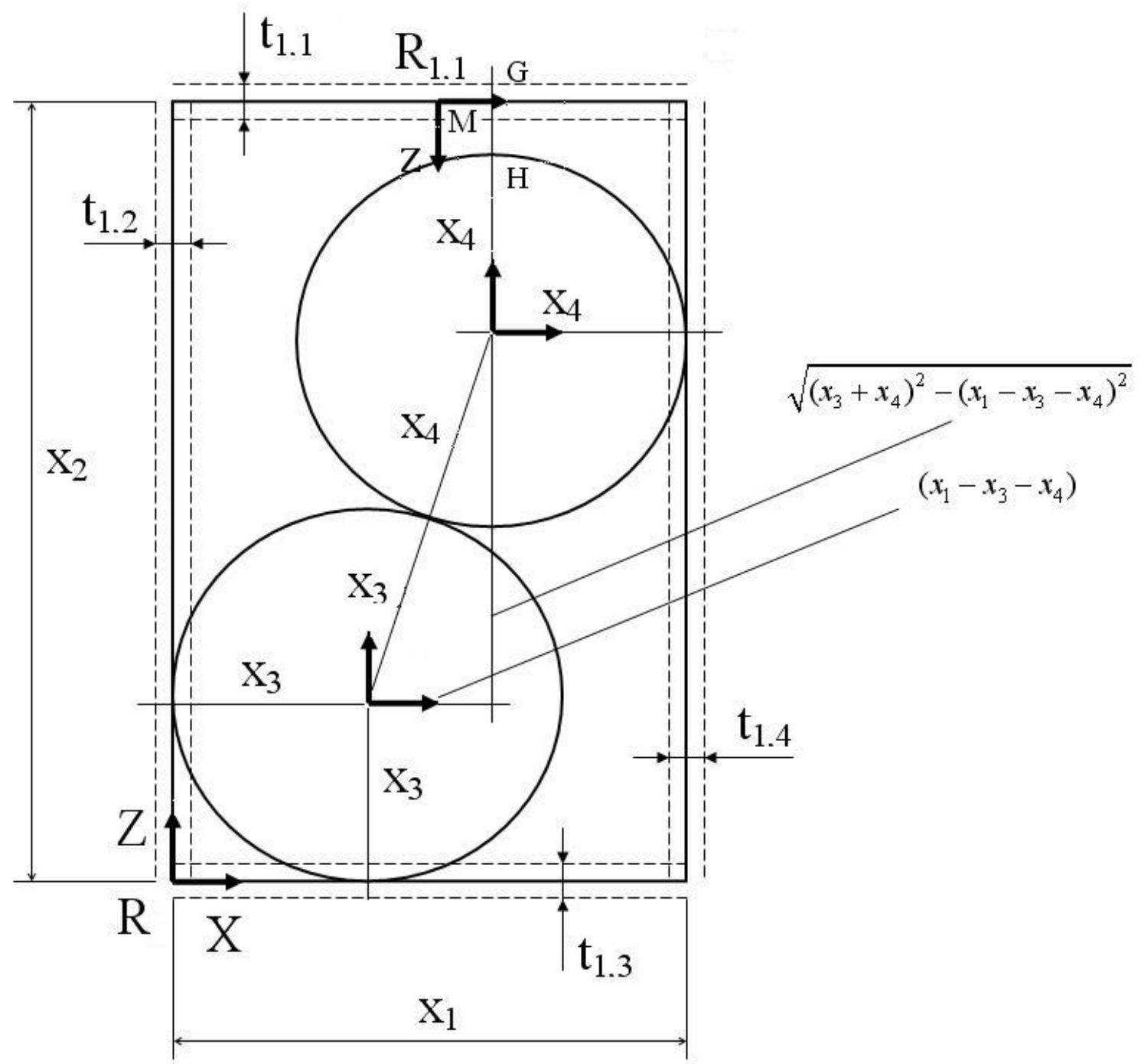

Figure 9 
Table 1

\begin{tabular}{|c|l|c|c|}
\hline \multicolumn{2}{|c|}{$\Delta \mathbf{g}$} & \multicolumn{2}{c|}{ Tolerance case } \\
\hline Method & \multicolumn{1}{c|}{ Approach } & only dimensional & dim. \& geo. \\
\hline exact geometric solution & worst case & $+0.84 \mathrm{~mm}$ & - \\
& & $-0.78 \mathrm{~mm}$ & \\
\hline \multirow{2}{*}{ jacobian } & worst case & $\pm 0.78 \mathrm{~mm}$ & $\pm 0.78 \mathrm{~mm}$ \\
\cline { 2 - 4 } & statistical case & $\pm 0.53 \mathrm{~mm}$ & $\pm 0.53 \mathrm{~mm}$ \\
\hline \multirow{2}{*}{ torsor } & worst case & $\pm 0.78 \mathrm{~mm}$ & $\pm 0.78 \mathrm{~mm}$ \\
\cline { 2 - 4 } & statistical case & - & - \\
\hline
\end{tabular}




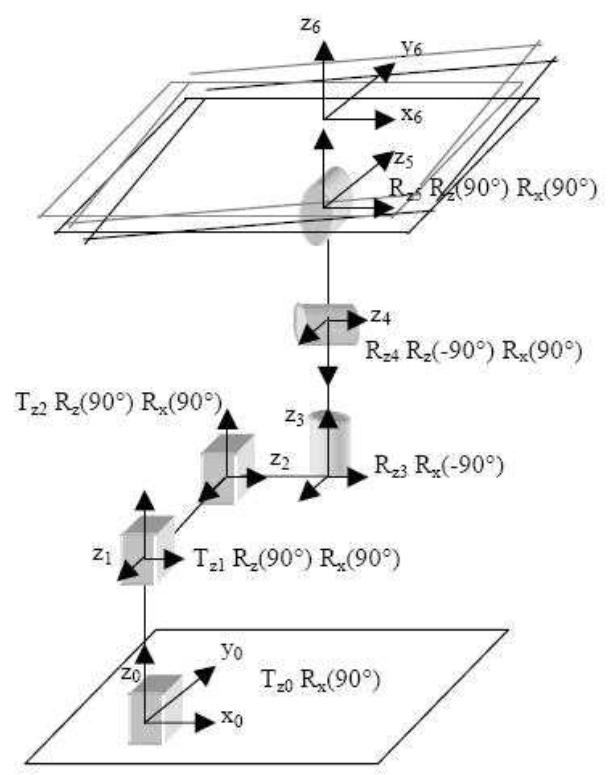

Figure 1

$199 \times 256 \mathrm{~mm}(96 \times 96 \mathrm{DPI})$

URL: http://mc.manuscriptcentral.com/tandf/tcim Email:ijcim@bath.ac.uk 


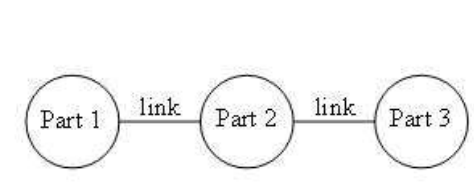

a)

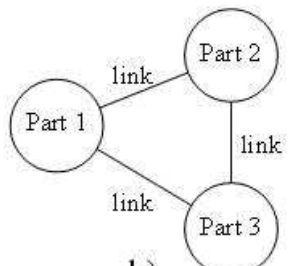

b)

Figure 2

$199 \times 256 \mathrm{~mm}(96 \times 96 \mathrm{DPI})$

URL: http://mc.manuscriptcentral.com/tandf/tcim Email:ijcim@bath.ac.uk 


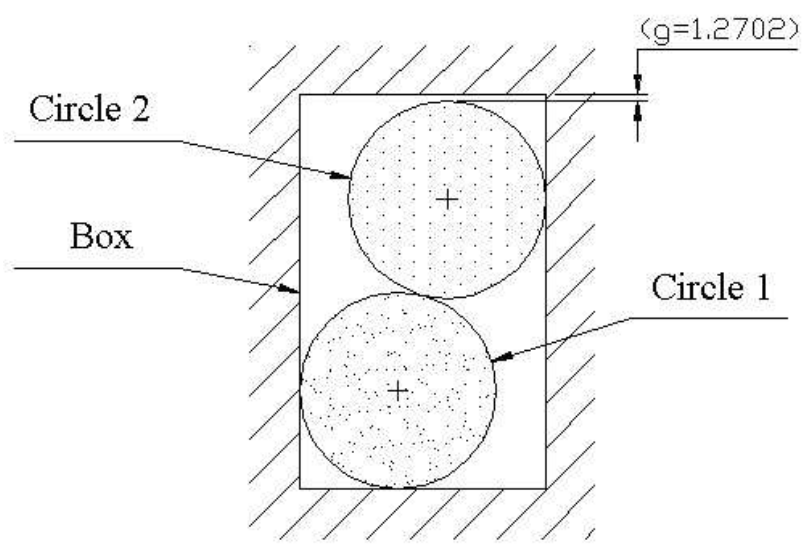

Figure 3

$199 \times 256 \mathrm{~mm}(96 \times 96 \mathrm{DPI})$ 


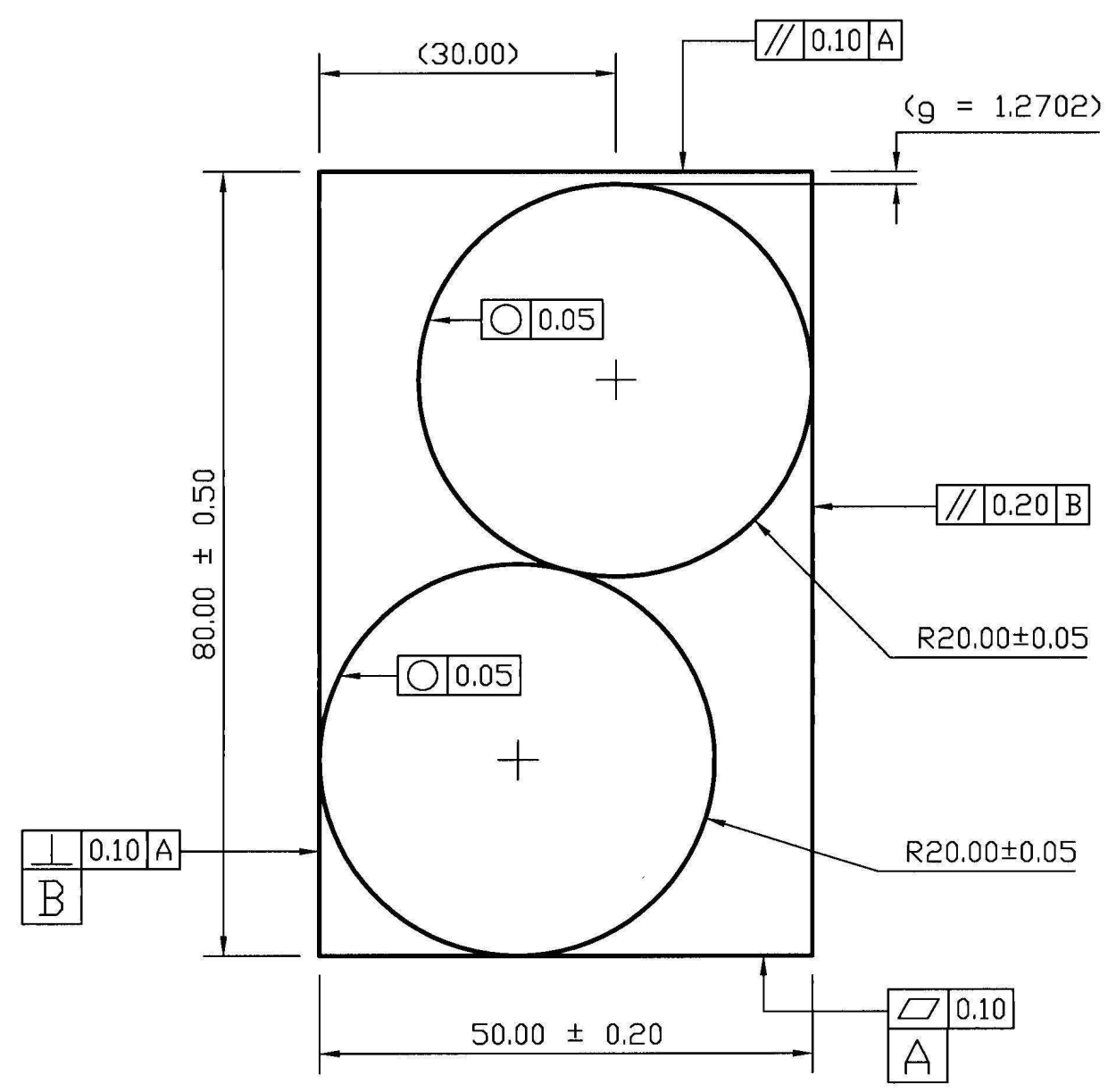

Figure 4

$524 \times 508 \mathrm{~mm}(96 \times 96$ DPI) 


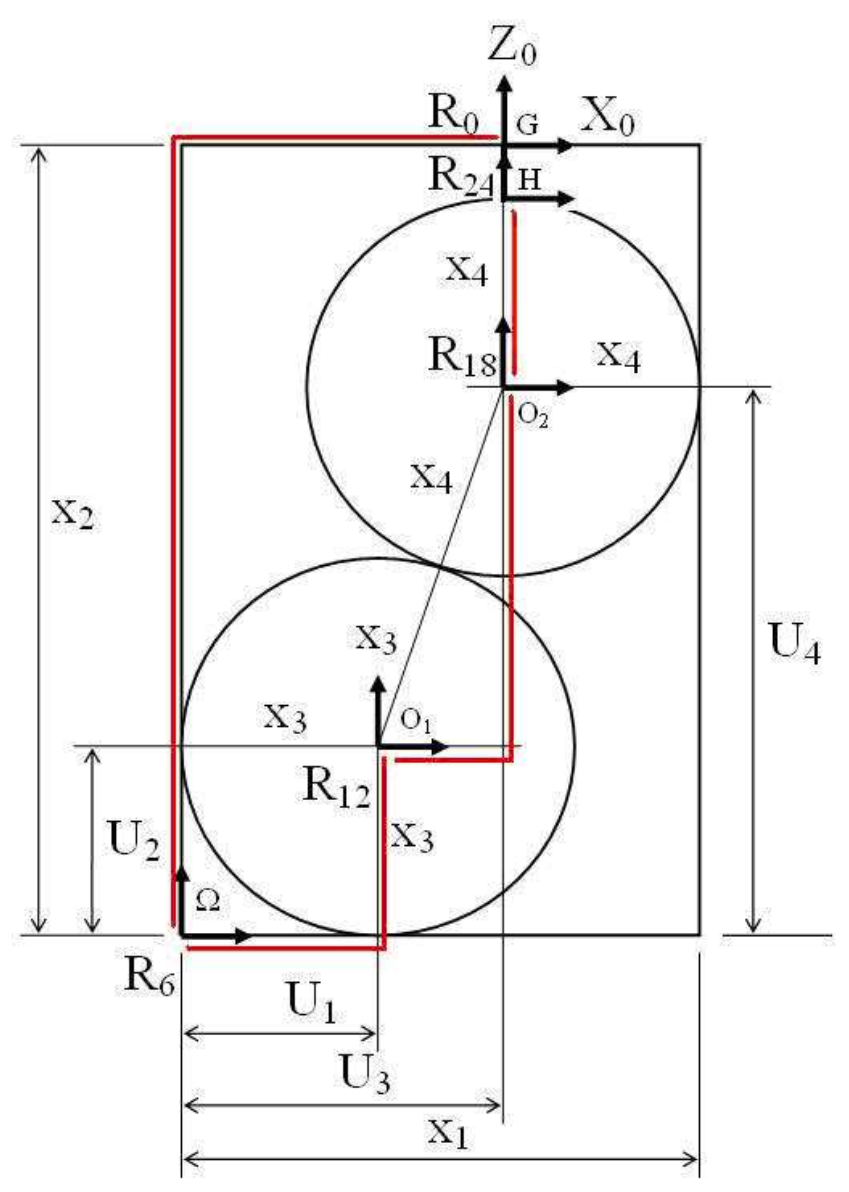

Figure 5

$199 \times 256 \mathrm{~mm}(96 \times 96$ DPI $)$ 


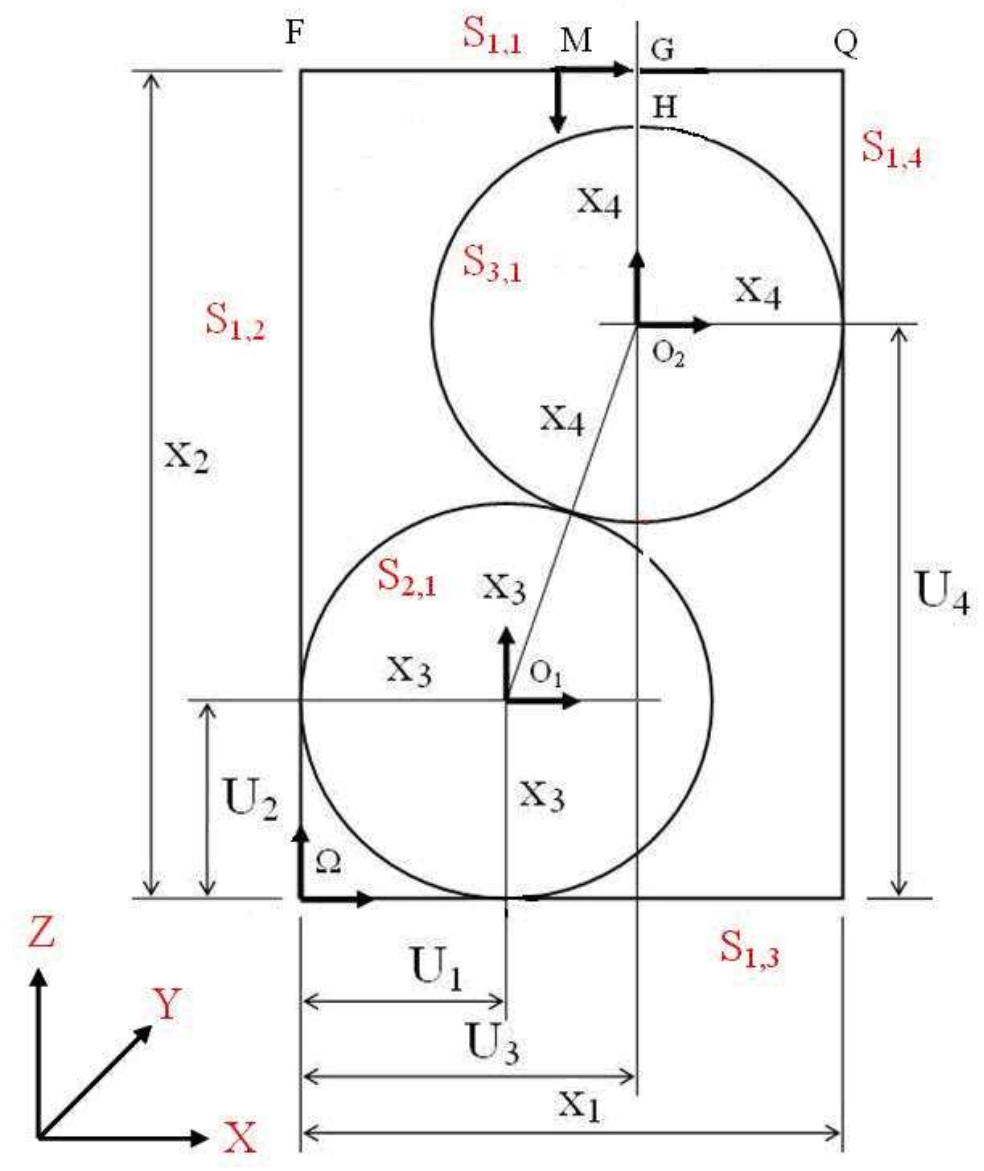

Figure 6

$199 \times 256 \mathrm{~mm}(96 \times 96$ DPI $)$ 


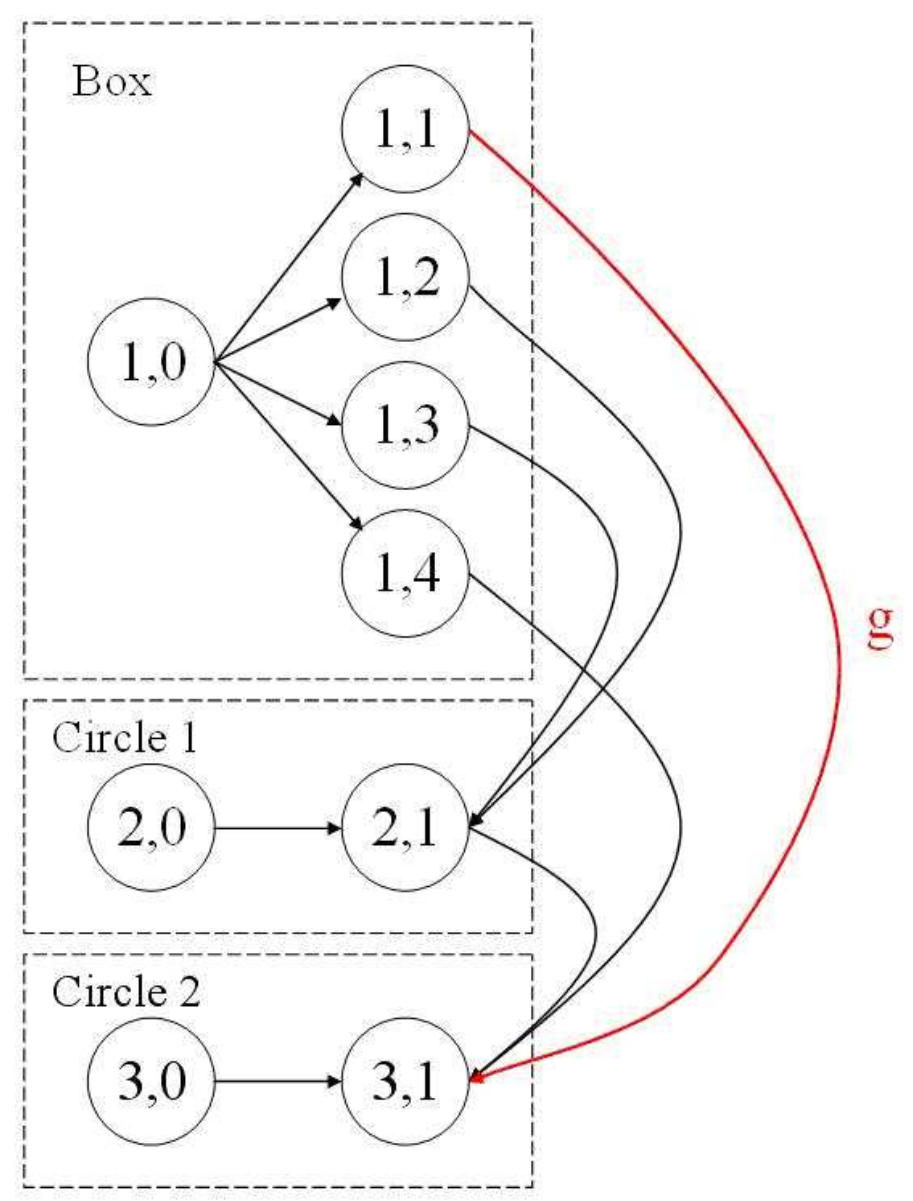

Figure 7

199×256mm (96 x 96 DPI) 


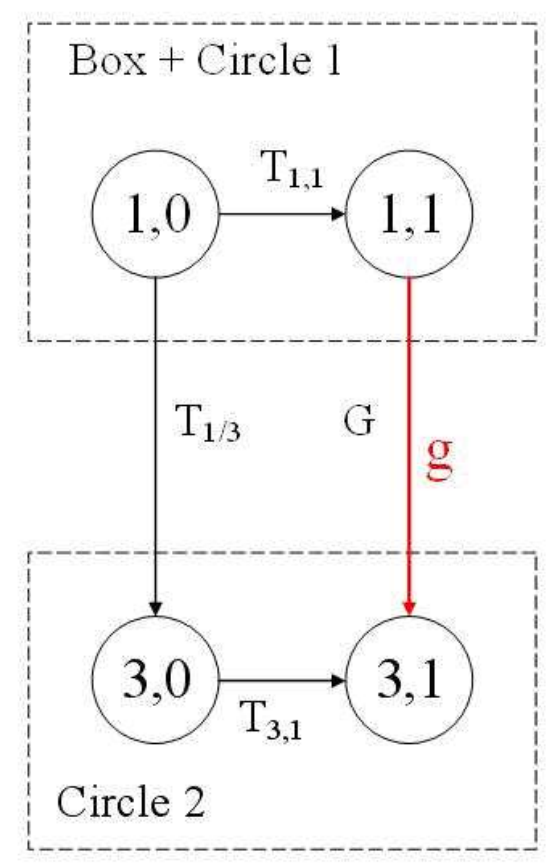

Figure 8

$199 \times 256 \mathrm{~mm}(96 \times 96 \mathrm{DPI})$ 


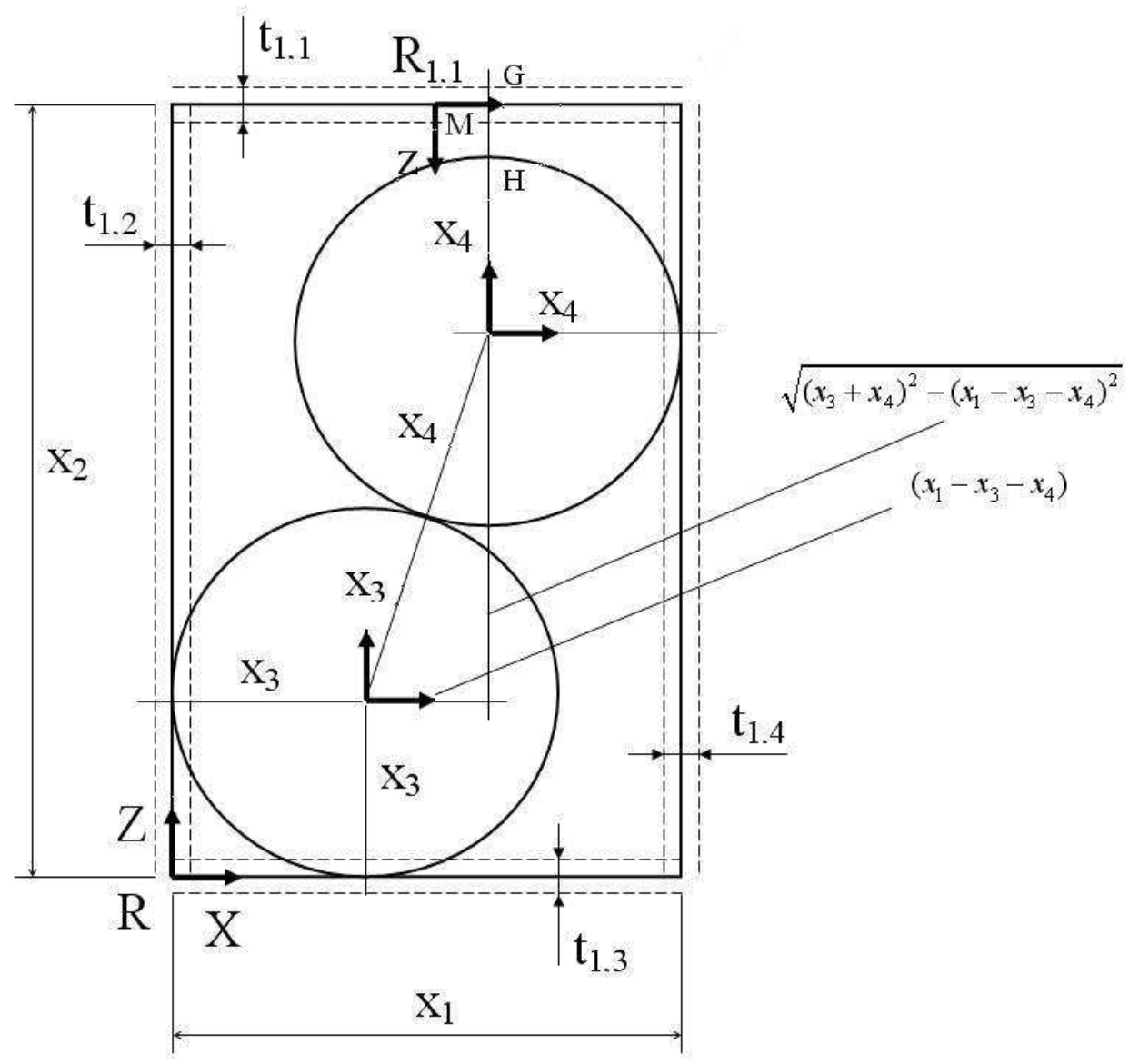

Figure9

$210 \times 256 \mathrm{~mm}(96 \times 96 \mathrm{DPI})$ 